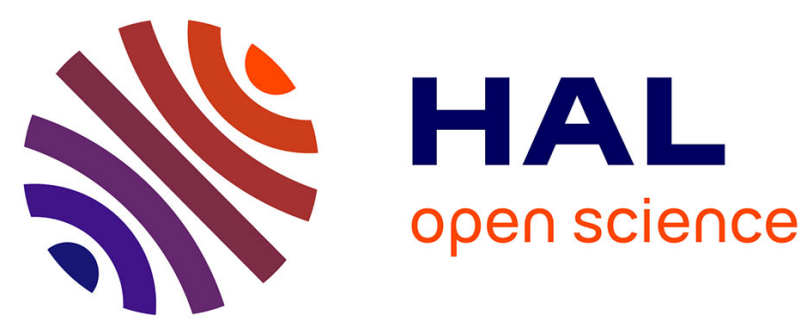

\title{
Le web matrimonial des migrants
}

Dana Diminescu, Matthieu Renault, Mathieu Jacomy, Christophe d'Iribarne

\section{To cite this version:}

Dana Diminescu, Matthieu Renault, Mathieu Jacomy, Christophe d'Iribarne. Le web matrimonial des migrants: L'économie du profilage au service d'une nouvelle forme de commerce ethnique. Réseaux: communication, technologie, société, 2010, 1 (159), pp.15-56. 10.3917/res.159.0015 . hal-02005546

\section{HAL Id: hal-02005546 \\ https://hal-sciencespo.archives-ouvertes.fr/hal-02005546}

Submitted on 4 Feb 2019

HAL is a multi-disciplinary open access archive for the deposit and dissemination of scientific research documents, whether they are published or not. The documents may come from teaching and research institutions in France or abroad, or from public or private research centers.
L'archive ouverte pluridisciplinaire HAL, est destinée au dépôt et à la diffusion de documents scientifiques de niveau recherche, publiés ou non, émanant des établissements d'enseignement et de recherche français ou étrangers, des laboratoires publics ou privés.

\section{(ㅇ)(1) 80}

Distributed under a Creative Commons Attribution - NonCommercial - ShareAlikel 4.0 


\section{DOSSIER}

\section{Les migrants connectés}

T.I.C., mobilités et migrations 


\title{
LE WEB MATRIMONIAL DES MIGRANTS
}

\author{
L'économie du profilage au service \\ d'une nouvelle forme de commerce ethnique
}

\author{
Dana DIMINESCU \\ Matthieu RENAULT \\ Mathieu JACOMY \\ Christophe D'IRIBARNE
}




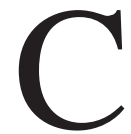

ommuniquer pour ne pas être déraciné, transporter sa sphère intime dans les espaces de ses migrations, faire travailler son réseau, sont des pratiques quotidiennes du migrant connecté. Ce « savoir se rencontrer » (qui a toujours été un savoir-faire indispensable à la reproduction identitaire et à l'intégration des migrants) s'est développé et diversifié en se manifestant sur le web. L'investissement des plates-formes sociales par les migrants est conséquent et ne pouvait pas se soustraire au domaine des relations intimes.

Les rencontres amoureuses en ligne ont fait durant ces dernières années l'objet de recherches nombreuses, par Eva Illouz (2006), Pascal Lardellier (2005), Serge Tisseron (2008), etc. On ne s’est néanmoins que très peu intéressé à ces espaces sur le web où la problématique de la rencontre rejoint celle des migrations et du mariage. Certes, ce terrain de recherche relève de l'univers du web amoureux : des technologies, des modes d'interaction, des pratiques similaires du web y sont à l'œuvre. Il n'en reste pas moins que des différences suffisamment sensibles dessinent un domaine spécifique. Ses frontières sont tout d'abord tracées par l'intention ou la finalité qui préside à la rencontre. En effet, le projet des individus inscrits est très souvent un projet de mariage, de telle manière qu'il faut parler d'un web matrimonial, dans lequel la tradition et les enjeux familiaux et communautaires jouent un rôle prépondérant. De plus, il peut également s'agir d'un projet de migration qui engendre de nouvelles formes de déplacement, de regroupement familial, peu étudiées à ce jour ${ }^{1}$. Ce terrain de recherche peut se nourrir des apports de nombreuses disciplines et spécialités : études migratoires, sociologie et histoire de la famille, cultural et gender studies, économie, analyse des réseaux et « web science ».

Nombreuses sont les raisons qui, selon nous, font du web matrimonial des migrants un domaine d'élection. En effet, il nous permet de penser les origines du web social. Si l'on en croit la frise chronologique proposée par Danah Boyd et Nicole Ellison et retraçant l'histoire des réseaux sociaux en ligne

1. Précisons dès à présent que le web matrimonial des migrants est fréquemment instrumentalisé pour la conclusion de mariages blancs. 
(2007), les origines du « réseautage social » sont à rechercher dans le développement (entre 1997 et 2001) de nombreux sites communautaires /ethniques / nationaux ayant offert des fonctionnalités de création de profils, gestion de liens d'amitié, etc. ${ }^{2}$ : AsianAvenue (Asiatiques-Américains), BlackPlanet (Africains-Américains), LunarStorm (Suédois), MiGente (Hispano-Américains), Cyworld (Sud-Coréens).

Quant au web amoureux, il constitue une seconde source décisive du web social. Rappelons que Friendster, premier réseau social à avoir acquis une forte popularité a été à l'origine conçu pour devenir le concurrent du site de rencontre Match, autrement dit pour investir le domaine des rencontres en ligne : l'hypothèse émise était que la probabilité que l'ami(e) d'un(e) ami(e) soit un bon partenaire était plus grande que pour un parfait inconnu ; le réseau, rappelle Boyd (2004), était ouvert aux non-prétendants à une rencontre dans la mesure où ceux-ci pouvaient avoir des amis recherchant l'« âme sœur » et ainsi jouer un rôle de connexion et de recommandation. Plus encore, les sites de rencontre " classiques » eux-mêmes peuvent être considérés comme des échantillons de la classe des réseaux sociaux, formant une catégorie particulière de ces plates-formes, relevant de ce que Dominique Cardon (2008) appelle le modèle du paravent : "Les individus se sélectionnent les uns les autres à travers une fiche critérielle découverte à l'aide d'un moteur de recherche, avant de dévoiler progressivement leurs identités et de favoriser une rencontre dans la vie réelle. » C'est ce que nous illustrerons plus loin à l'aide d'exemples concrets.

Dans cet article, nous ferons état des premiers questionnements issus de l'exploration du web matrimonial des migrants. C'est autour de trois problématiques, distinctes mais étroitement liées, que s'articule notre recherche :

- Il s'agit tout d'abord de rendre compte de l'économie à l'œuvre dans le web matrimonial des migrants (WMM dans la suite de cet article) : c'est une économie des bases de données fondées sur le profilage communautaire/ethnique des candidats à la rencontre/mariage, le «matching » des profils et leur dépassement dans la communication (électronique, téléphonique) et la rencontre " en chair et en os ». Comment cette chaîne socio-technique, fondée sur une certaine marchandisation des identités, se déploie-t-elle au sein de différentes communautés de migrants?

2. Le site " généraliste » sixDegrees.com (lancé en 1997 et abandonné en 2001), authentique précurseur des fameux MySpace et Facebook est une exception. 
- Le WMM est le lieu d'une articulation complexe du genre, de la nationalité et de la mobilité. Il révèle en premier lieu ce que l'on appellera une distribution internationale du genre au sens de variations importantes de la répartition hommes/femmes selon les nationalités sur les différentes plates-formes matrimoniales. Cette distribution est régulièrement liée à une " circulation » intercommunautaire online. Elle est par ailleurs corrélative des « écarts » dans le désir de migration selon les communautés et selon les genres.

- Enfin le WMM interroge la notion de "commerce ethnique " (ethnic business) dans la mesure où ce dernier s'appuie sur des réseaux ethniques préexistants, il active ces réseaux tandis que, dans le WMM, c'est la « mise en relation » d'individus non liés qui est le moteur de l'économie en question. Le WMM pose l'équation : faire du réseau = faire du commerce. Ainsi, l'on peut se demander s'il ne constitue pas une forme inédite de commerce ethnique.

\section{Trois études de cas - une méthodologie mixte}

Notre recherche repose sur trois études de cas (Diminescu, Renault, Gangloff, Picard ; d'Iribarne, Hassane, 2009) : la première est consacrée à l'offre de sites de rencontre communautaires - sur des critères d'affinité ethnique, sexuelle, physique, etc. - proposés par la société australienne Cupid $\mathrm{Media}^{3}$; la seconde porte sur le mariage mixte franco-« pays de l'Est », notamment par l'entremise de l'agence online/offline Eurochallenges ${ }^{4}$. La troisième s'intéresse au mariage intracommunautaire au sein de la diaspora indienne via le site Shaadi ${ }^{5}$. Nous avons ainsi délibérément opté pour trois terrains présentant des différences importantes du point de vue des stratégies matrimoniales mises en œuvre, des motivations et critères de sélection du potentiel conjoint, du rôle de l'identité et de la différence (ethnique, culturelle, etc.) dans l'union, des fonctions de l'homophilie et de l'hétérophilie.

Notre recherche s'appuie sur un dispositif de fouille de l'internet qui nous a permis de construire différents types de corpus : par collecte semi-automatique de sites web, par aspiration automatique de profils, par extraction de " success stories ", par inventaire de services matrimoniaux (s'agrégeant autour de l'économie du profilage). L'analyse de profils constitue le cœur de nos investigations. Or l'accès aux profils sur les sites requiert de compléter

3. http://www.cupidmedia.com/

4. http://www.eurochallenges.com/

5. http://www.shaadi.com/. Cette étude de cas a été réalisée en collaboration avec Souley Hassane, chercheur au laboratoire Migrinter, Poitiers. 
des formulaires (pour la recherche et éventuellement pour l'inscription) permettant d'interroger la majorité des contenus proposés. Pour cette raison, ces sites appartiennent au web profond (deep web), peu accessible aux moteurs de recherches. C'est pourquoi nous avons développé des heuristiques spécifiques à chacun de ces sites, c'est-à-dire des séries d'instructions pour l'extraction de données ciblées ${ }^{6}$; ces données de profils, stockées dans un tableur, autorisent dès lors des analyses statistiques ${ }^{7}$. Enfin, nous avons créé deux profils fictifs sur deux sites de Cupid Media afin d'étudier les profils et les messages des membres des sites ayant témoigné de leur intérêt. L'ensemble de ces expérimentations a répondu au souci du respect déontologique de l’anonymat.

\section{UNE ÉCONOMIE DU PROFILAGE ETHNIQUE}

\section{Le web amoureux : une économie du profil et de l'« au-delà » du profil}

Le web amoureux - et a fortiori le WMM - se situe à l'intersection d'une économie participative et d'une économie de services. Plus précisément, il met « au service » d'une économie de services la participation de réseaux de clients. C'est la profonde intrication de ces deux économies qui expose irrémédiablement l'amour et le mariage médiés par le web aux technologies $\mathrm{R}$ (technologies relationnelles) et en particulier aux techniques du profilage des utilisateurs (user profiling). Ce profilage n'est pas un «à-côté » de l'usage que l'internaute ignorerait mais le cœur même du service proposé : l'utilisateur en est l'agent dans la mesure où c'est du renseignement adéquat des champs du profil que dépend un bon matching, c'est-à-dire la possibilité d’une complémentarité entre les prétendants à la rencontre amoureuse (Illouz, 2006) ${ }^{8}$.

6. Ces heuristiques sont intégrées et mises en œuvre par le logiciel Navicrawler. Ce dernier est une extension pour le navigateur web Firefox, développée par Mathieu Jacomy ; c'est un outil d'exploration du web permettant d'en analyser les contenus et la structure. Il s'agit d'un logiciel libre, téléchargeable à l'adresse suivante : http://webatlas.fr.

7. Nous avons ainsi pu aspirer 600 profils ( 300 hommes et 300 femmes) pour trois sites de la gamme Cupid Media (www.filipinaheart.com, www.mexicancupid.com, www.russianeuro. com) et 18000 profils pour un quatrième, le plus important : www.muslima.com. Pour Eurochallenges, nous nous sommes limités aux profils de femmes de nationalité russe, soit environ 1600 profils. Enfin, pour Shaadi, nous avons pu recueillir 9000 profils (4500 hommes et 4500 femmes) d'Indien(ne)s vivant en Inde et dans les pays d'émigration.

8. On ne s'étonnera pas dès lors que les sites du web amoureux possèdent la structure d'un marché : « les sites de rencontre ont introduit dans le domaine de la rencontre amoureuse les principes fondamentaux de la consommation de masse - l'abondance, la liberté de choix, l'efficacité, la rationalisation, le ciblage sélectif et la standardisation ». 


\section{Figure 1. Extrait d'un tableau de profils du site Shaadi}

\begin{tabular}{|c|c|c|c|c|c|c|c|}
\hline Age Mantal Status & Chiddren & Height & Complevion & Manglik/KujaDosham & Special & Case Blood Group & Education \\
\hline 31 Never Married & No & $5^{\prime} 5^{\prime \prime}$ & Fair & Not applicable & None & 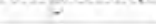 & Diploma - Management \\
\hline 42 Separated & No & $52^{m}$ & Fati & Don't Know & None & - & Bachelors - Lav \\
\hline 31 Never Married & No & $5 \mathrm{~B}^{\mathrm{\prime \prime}}$ & Fair & Not applicable & None & - & Diploma - Shipping \\
\hline ao Never Marned & No & $5.5^{m}$ & Fair & Don't know & None & - & Eacheiors - Commerce \\
\hline $2 \mathrm{~B}$ Never Mamed & No & 5. $\mathrm{s}^{* 2}$ & Farr & Don't know & None & - & Masters - Commerce \\
\hline 47 Divorced & Yes. Not living together & $57^{4 k}$ & Farr & Don't Know & None & At & High school - Administratrve \\
\hline 21 Never Mamed & No & (5. $4^{21}$ & Farr & Don't Know & None & + & Bacheiors - Medicine \\
\hline 29 Never Mamed & No & $56^{*}$ & Fair & Don't know & None & o+ & Masters - 5cience \\
\hline 52 Divorced & Yes. Lwing together & (5) $4^{\text {wn }}$ & Farr & Don't Know & None & Dontt know & Diploma \\
\hline 35 Never Marned & No & $154^{m=}$ & Fair & Don't know & None & c & Masters - Mursing/ Heatth 5 \\
\hline 25 Never Married & No & $53^{n=}$ & Fair & Not applicable & None & $=$ & Bachelors - Engrneering/ Tf \\
\hline 34 Divorced & Yes. Luving together & $55^{*}$ & Fair & No & None & A+ & Associates degree - Finand \\
\hline 29 Never Marned & no & $50^{4 n}$ & Fair & Not applicable & None & - & Duploma - Commerce \\
\hline 32 Never Marned & No & $5 \mathrm{~B}^{-}$ & Fair & Not applicable & None & - & Diploma - Management \\
\hline 52 Never Marned & No & $4^{4} 11^{\prime \prime}$ & Fair & Not applicable & None & - & Honouirs degree - Finance \\
\hline 32 Separated & Ves. Living together & $55^{\circ}$ & Fair & Don't know & None & - & Honours degree - Compute: \\
\hline 40 Never Marned & No & $155=$ & Fair & Don't know. & None & - & Bachelors - Medicine \\
\hline 44 Widowed & No & $59^{\prime \prime}$ & Fair & Not applicable & None & $=$ & Diploma - Management \\
\hline 25 Never Marned & No & $55^{\circ}$ & Fair & Don't know & None & Don't know & Bachelors - Management \\
\hline 2ânever Marned & No & $51=$ & Fair & Don't Know & None & $\mathrm{AB}+$ & Diploma - Administrative se \\
\hline 45 Divorced & Yes. Not living together & $51^{\pi / 1}$ & Fair & Yes & None & - & High school \\
\hline 44 Widowed & Yes. Uiving together & $5^{5} 4^{*}$ & Fair & Don't Know & None & - & Bachelors - Nursing/ Health \\
\hline 35 Never Marned & No & $50^{\mathrm{mm}}$ & Fair & Don't know & None & - & Honours degree - Science \\
\hline 21 Never Marnied & No & $6^{\prime} 11^{t h}$ & Fair & Don't Know & None & $0+$ & Trade school - Arts \\
\hline 33 Never Marned & No & $410^{\prime 2}$ & Fair & Don't know & None & 5 & Bachelors - commerce \\
\hline 30 Divorced & No & 52 & Fair & Don't know & None & - & Bachelors - Finance \\
\hline 45 Divorced & res. Living together & $50^{\prime \prime \prime}$ & Fair & Don't know & None & A+ & Less than high school \\
\hline 24 Never Marned & No & $54^{n i n}$ & Fair & Don't know & None & Don't know & Bachelors - Arts \\
\hline 23 Separated & Yes. Living together & $56^{\prime \prime \prime}$ & Fair & Don't know & None & . & Diploma - Finance \\
\hline 23 Never Marned & No & $5^{1} 2^{\prime \prime}$ & Fair & Don't know & None & $\mathrm{A}+$ & Bachelors - Commerce \\
\hline a1 Never Marned & No & $52^{-1}$ & Fair & Don't know & None & c. & Honours degree \\
\hline 30 Nhever Marned & No & $4^{\prime} 10^{\prime \prime}$ & Fair & Don't Know & None & - & Diploma \\
\hline 33 Never Marned & No & $50^{\circ \prime \prime}$ & Fair & Not applicable & None & - & Bachelors \\
\hline 36 Never Marned & No & $5^{1} 3^{-1}$ & Farr & Not applicable & None & - & Diploma - Managenvent \\
\hline 34 Never Marned & No & $500^{\mathrm{mm}}$ & Fair & Don't Know & None & - & Bachelors \\
\hline 28 Never Marned & No & $5,2^{\prime \prime}$ & Fair & Don't Know & None & $=$ & High school - Finance \\
\hline 29 Divorced & No & $5^{\prime} 5^{\circ}$ & Fair & Don't know & None & $0+$ & Masters - Science \\
\hline 34 Divorced & No & $5^{\prime} 3^{-\infty}$ & Fair & No & None & Don't know & Doctorate - Science \\
\hline 40 Never Marned & No & 5170 & Fair & Not applicable & None & 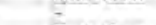 & Honours degree - Managem \\
\hline 36 Neever Marned & No & $5^{\prime} 5^{*}$ & Fair & Don't Know & None & Don't know & Masters - Arts \\
\hline 42 Divorced & Yes. Living together & $52^{\prime \prime}$ & Fav & Don't Know & None & Don't know & Diploma - Management \\
\hline Jo Never Marned & No & $54^{* \prime \prime}$ & Far & Not applicable & None & - & Diploma - Finance \\
\hline 32 oivorced & No & $5^{\prime} 2^{2}$ & Fair & Don't know & None & 日+ & Undergraduates - commerco \\
\hline 20 oivorced & No & $5^{\prime} 5^{\prime \prime \prime}$ & Faur & Don't know & None & - & Less than hogh school \\
\hline 31 Newer Marned. & No & $5^{\prime} 5^{\prime \prime \prime}$ & Far & Don't know & None & Dorn't know & Masters - Computers/ IT \\
\hline 37 Neover Marned & No & $5^{\prime} 00^{\prime \prime \prime}$ & Fas & Dorit know & None & 2 & Bachelors - Fine arts \\
\hline
\end{tabular}

On objectera peut-être que ceci n'est pas propre au web amoureux et vaut pour le web social en général, en particulier pour les réseaux sociaux en ligne sur lesquels les individus renseignent leurs profils, divulguent, diffusent des informations personnelles, s'exposent. Ainsi que 1'écrit Karine Douplitzki (2009), "plus qu'être anonyme, ce qui compte c'est être visible ! ". Cependant, l'usage commercial des données dévoilées sur les réseaux sociaux est soit inconnu de l'utilisateur, soit la contrepartie acceptée des services proposés. Au contraire, dans le web amoureux, l'individu participe pleinement à la marchandisation du profil (qui n'est rien d'autre que le service offert luimême) : il paie pour être vu et pour voir les autres.

Les sites du web amoureux fonctionnent sur le modèle de l'abonnement pour une période déterminée, présentée comme une durée raisonnable pour rencontrer l'« âme sœur ». De nombreux sites proposent une série d'abonnements dont le coût est proportionnel à l'étendue des fonctionnalités disponibles. 
« Payer plus » - et tel est l'argument de vente - signifie maximiser les probabilités de rencontre en offrant des possibilités de se libérer du profil. En effet, " payer plus » équivaut à échapper à la standardisation en multipliant les modalités d'exposition de soi et de communication : ajouter des photos et vidéos au profil, décrire en texte libre « un profil de personnalité », communiquer en messagerie instantanée, etc. Ainsi faut-il noter que l'économie du profilage est tout autant fondée sur l'accès aux bases de profils que sur la sortie hors des profils vers ce qui deviendra (peut-être !) communication par messagerie électronique ou messagerie instantanée sur le site, puis hors du site (adresses personnelles), communication téléphonique et enfin rencontre physique.

Le profil en tant que contenu ne devient donc marchandise que dans la mesure où il est à la fois ce qui rend possible et ce qui limite la rencontre et demande ainsi à être dépassé : c'est une économie du profil et de l'« au-delà » du profil.

Il nous faut à présent ajouter d'une part que le principe du paravent, du voilement/dévoilement, décrit par Dominique Cardon, ne concerne pas seulement la recherche et le matching de profils mais aussi la sortie hors du cadre catégoriel du profil ; il se rapporte aux individus « en chair et en os » et est un excellent descripteur de la rencontre dans son ensemble. D'autre part, c'est un principe proprement économique puisque c'est ce jeu de l'exposition et de la dissimulation qui constitue la source de valeur marchande. De tout cela, on retiendra que l'économie du profilage ne se limite en aucun cas aux préliminaires de la rencontre (recherche de profils) mais qu'elle tend à incorporer toutes les étapes de la rencontre amoureuse.

Le WMM ne déroge pas à la règle. Ainsi sur les sites de la société Cupid Media, sont proposés plusieurs abonnements hiérarchisés : standard, gold, platinum (modèle de la carte bleue). Plus le prix est élevé, plus les opportunités de sortie du profil sont nombreuses (Pissard, 2009) ${ }^{9}$. Quant aux enjeux d'une économie de $l^{\prime}$ « au-delà » du profil, ils sont clairement identifiables dans la success story suivante que nous résumons :

9. Plus également il élargit le champ des partenaires potentiels, puisque « l'utilisateur payant» est autorisé à lire les messages des partenaires ayant un abonnement gratuit. Ce modèle permet de faire coexister le « payant » et le « gratuit » et valorise nécessairement les utilisateurs payants, jugés « plus motivés » et « plus sérieux » que les autres. On notera qu’il est particulièrement adapté au positionnement de niche, donnant le sentiment à l'utilisateur d'« être chez soi ». 
Rafique et Dayu se sont rencontrés sur le site Muslima. Dayu avait souscrit à un abonnement gold sur le site. Rafique, qui de son côté avait un abonnement standard, lui envoya un message. Le réflexe de Dayu fut alors de consulter le profil de Rafique, profil incomplet, sans photographies attachées, peu engageant. Elle décide tout de même de lui répondre. Puis, ils échangent leurs numéros de téléphone et commencent à parler ensemble durant les soirées, partageant leurs expériences, leurs intérêts, etc. Aux communications téléphoniques s'ajoutent les messages électroniques et les sms. Dayu en vient à collecter tous les sms dans un album. À peine plus d'un mois plus tard, ils s’avouent réciproquement leur amour puis en viennent à décider de se marier quatre mois plus tard. Dayu avoue alors qu'ils n'avaient plus le temps pour se rencontrer ou « aller au cinéma » mais qu'ils communiquaient quotidiennement par téléphone et sms. Enfin, Dayu remercie Allah et Muslima pour ce qui est, dit-elle, un « rêve devenu réalité ». ${ }^{10}$

Cette histoire, mise en exergue sur www.muslima.com, a évidemment une fonction publicitaire ${ }^{11}$; elle doit prouver que de multiples configurations (différents abonnements, etc.), même les plus défavorables en apparence (le profil de Rafique est très pauvre), sont susceptibles de conduire à une rencontre ou à un mariage réussi. Il n'en reste pas moins que l'histoire dévoile bien le dépassement du profil vers des formes de communication offrant plus de possibilités d'exposition de soi à l'autre.

\section{Un profilage ethnique - la communautarisation de la rencontre}

La question décisive que pose le WMM est celle du rôle que joue l'ethnicité (la religion, le « pays d'origine ») dans l'économie du profilage. Dans quelle mesure peut-on parler d'un profilage ethnique qui ne serait plus tant un ensemble de mécanismes mis en œuvre par des autorités (le plus souvent étatiques) pour le contrôle, la gestion de leur population mais le produit même de pratiques d'ethnicisation par les individus eux-mêmes ${ }^{12}$.

10. http://www.muslima.com/french/Success.cfm.

11. La " success story » vise par ailleurs à transformer en modèle, voire en " business plan » des histoires singulières.

12. Il ne s'agira pas ici seulement de faire un diagnostic de l'homophilie dans le WMM. À vrai dire, celle-ci est aussi décisive sur un site généraliste comme Meetic. L'enjeu est bien plutôt d'observer les configurations dans lesquelles l'ethnicité devient le critère fondamental de l'homophilie (alors que, dans Meetic, on parlerait plutôt d'homophilie sociale, économique, professionnelle, etc.). 
Ce que nous laisse observer le web matrimonial des migrants, c'est d'abord une forte tendance à la communautarisation (au sens d'une division en communautés) de la rencontre et du mariage médiés par le web. Les grandes plates-formes communautaires - www.afrik.com (Afrique et Maghreb), www.bladi.net (Maroc), etc. - s'en font l'écho à travers des articles qui saluent la possibilité de rechercher l'âme sœur sur des critères culturels, ethniques, religieux ${ }^{13}$, en posant cependant la question du « risque de repli communautaire $"{ }^{14}$. L' " observatoire des sites de rencontre » Dating Watch souligne la tendance en France (à la suite des États-Unis) à la spécialisation, autrement dit au développement de sites de rencontre dédiés à des « catégories de population » minoritaires ${ }^{15}$. L'annuaire des sites de rencontre Ecupidon propose une rubrique de sites de rencontre par «affinités ethniques $»^{16}$. Des polémiques sont apparues à ce sujet sur les forums de sites féminins ou de psychologie ${ }^{17}$. Enfin, des régies publicitaires, telles que Publi Ethnik, s'emparent du phénomène en proposant des positionnements sur les principaux sites de rencontre communautaires en arguant du succès de ces derniers et de l'existence d'un véritable «marché parallèle au marché grand public, qui est un marché par communauté ${ }^{18}$.

13. http://www.bladi.net/forum/115343-amour-net-coeur-sites-rencontres-communautaires/

14. http://www.afrik.com/article6881.html.

15. Nos propres observations de terrain dans les cybercafés parisiens fréquentés par les migrants, ont dévoilé une tendance forte à la consultation des sites de rencontre.

16. Faisons ici remarquer qu'une exploration non exhaustive du web matrimonial et de rencontre maghrébin francophone nous a permis quant à nous de découvrir très rapidement douze sites proposant des services équivalents.

17. «Pour ma part, je trouve que l’idée de se “spécialiser” n’est pas bien. On fait la guerre contre le racisme et l'exclusion. Faire des recherches plus poussées pourquoi pas, mais de là à ne privilégier qu'une catégorie, je trouve ça dommage et ça limite le plaisir de connaitre d'autres cultures » http://forum.elle.fr/htm2/les-sites-de-rencontres-communautaires,d-8829. aspx. Voir également http://forum.doctissimo.fr/psychologie/celibat/communautaire-rencontrereaction-sujet_169574_1.htm.

18. http://www.publiethnik.com/ 
Figure 2. Quelques sites/marchés de la gamme Cupid Media

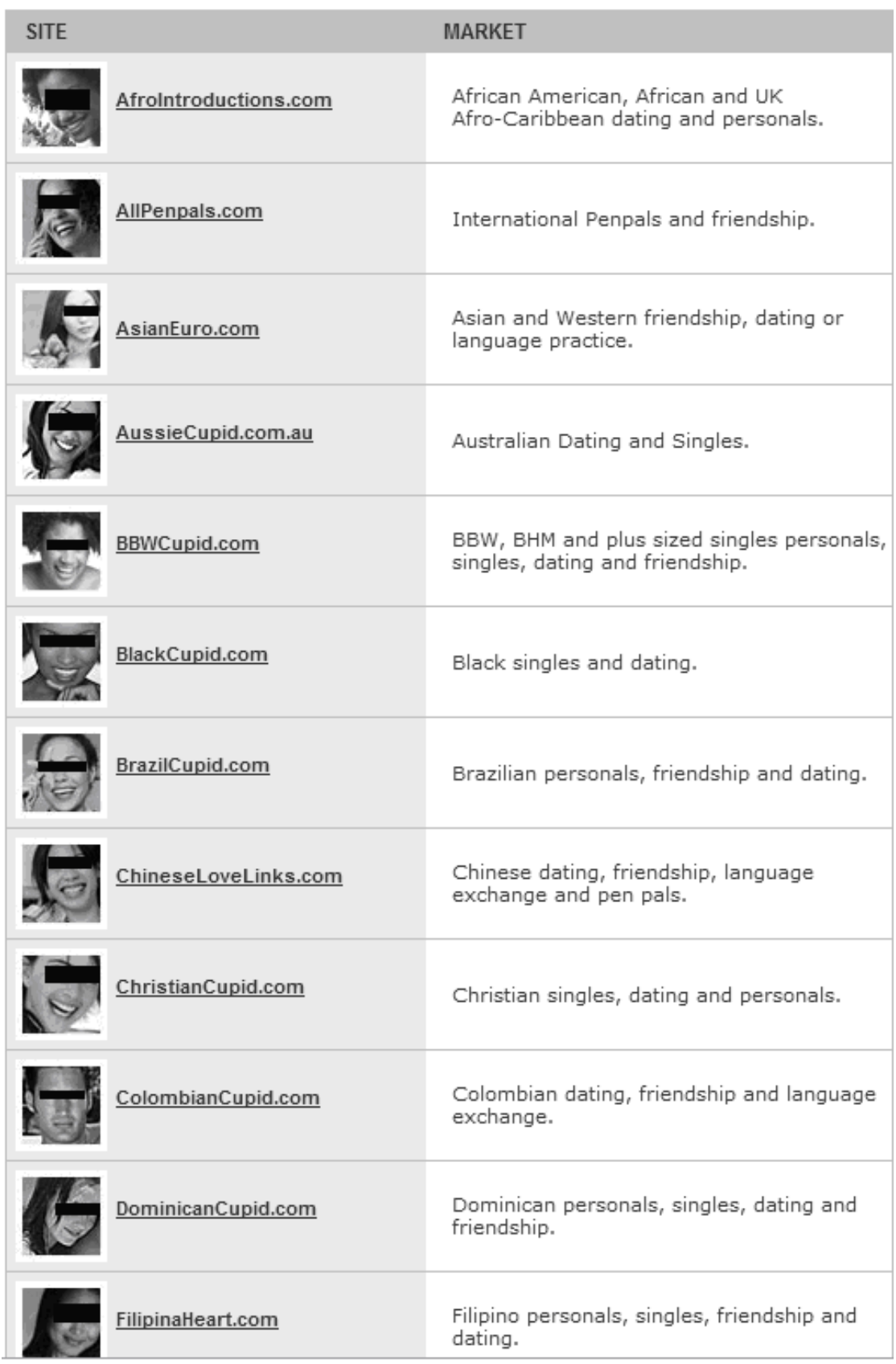


La société australienne Cupid Media a exploité cette niche économique. Sur son site, elle se présente comme « une société de services (...) qui gère des sites de rencontre de niche (...) basés sur l'ethnicité, l'apparence physique, la préférence sexuelle ou des intérêts spéciaux ${ }^{19}$. Cupid Media fournit par conséquent des instruments standards de gestion et d'accès à des bases de données de profils, déclinés pour plusieurs communautés nationales ou ethniques ${ }^{20}$ : BlackCupid.com, BrazilCupid.com, ChineseLoveLinks.com, etc. Chacune de ces communautés est considérée comme un « marché » (de niche).

Précisons que si cette division par communauté se matérialise ici à travers la création de plates-formes de rencontre distinctes pour chaque groupe, cela ne signifie bien sûr nullement que l'importance du critère ethnique serait nulle sur les sites de rencontre "généralistes ». Des recherches ont ainsi été menées aux États-Unis (Hitsch, Hortacsu, Ariely, 2005) sur un célèbre site de rencontre ${ }^{21}$. Sur celui-ci, les utilisateurs déclarent leur appartenance ethnique et peuvent aussi préciser si l'ethnicité du potentiel partenaire est un facteur important pour eux : $38 \%$ des femmes et $18 \%$ des hommes affirment ainsi vouloir rencontrer une personne appartenant au même groupe ethnique qu'eux (pour les «Caucasiens ", ces chiffres sont respectivement de $48 \%$ et $22 \%$; pour les « Noirs », de $25 \%$ et de $8 \%$ ). La différence en termes de genre est ainsi frappante. La préférence communautaire, affirment les auteurs, est plus forte chez les femmes que chez les hommes. Ce que dévoilent ici les profils est confirmé par les interactions entre utilisateurs : par exemple les hommes « AfricainsAméricains » et « Hispaniques » reçoivent environ moitié moins de messages provenant de femmes blanches que les hommes blancs - pour les « Asiatiques », c'est même quatre fois moins. Nous ne pouvons qu'en conclure que la discrimination n'est pas le seul fait des sites de rencontre communautaire et qu'elle opère également sur les sites généralistes. Il n'en reste pas moins que la «matérialisation » de la communautarisation ne fait probablement pas que reproduire des discriminations ; elle produit de l'ethnicité.

19. http://www.cupidmedia.com/ (traduit de l'anglais).

20. L'exemple de Muslima nous montre que la religion peut être un critère décisif. Ainsi Muslima est le site le plus populaire de la gamme Cupid avec 1204000 membres (23 mars 2009). Mais l'on retrouve également un site pour la communauté chrétienne : www.christiancupid. com qui affiche 67000 membres (23 mars 2009). Les critères ethniques/religieux ne sont pas les seuls critères communautaires. La préférence sexuelle (gays et lesbiennes), le statut familial (parents célibataires) ou encore le type de relations recherchées («penpals » - correspondants) sont ainsi des vecteurs d'affinités devant justifier l'existence de sites distincts.

21. Le nom du site n'est pas donné par les auteurs. 


\section{La standardisation des différences}

On peut par ailleurs se demander si c'est vraiment sur les différences entre communautés que se développe l'économie du profilage ethnique. En effet, nous avons vu que c'étaient les mêmes outils qui étaient déployés sur chacun des sites de rencontre communautaire, de telle manière que ces sites sont, dans leur structure (menus, formulaires de recherche, etc.), identiques. Plus encore, les différentes catégories qui composent les profils sont exactement les mêmes d'une communauté à l'autre : sexe, nationalité, ethnicité, emploi, etc. Autrement dit, loin de favoriser la reconnaissance et l'expression des différences ethniques/ culturelles, la communautarisation s'opère sous le signe de leur effacement, d'une extrême standardisation. Nous pourrions ainsi parler d'un degré zéro de la différence. La séparation des communautés ne s'explique pas par des besoins distincts et ne s'exprime pas sous la forme de fonctionnalités spécifiques à chacune d'entre elles ; elle est tout entière concentrée dans le nom même du site résumant la qualité identitaire/communautaire du public visé. Les différences sont, d'une certaine manière, rendues plus visibles par le fait même de se détacher sur un fond commun, sur une structure unique. Les deux profils suivants illustrent cette standardisation - trouver des différences y est une gageure :

Figure 3. Un profil sur www.muslima.com

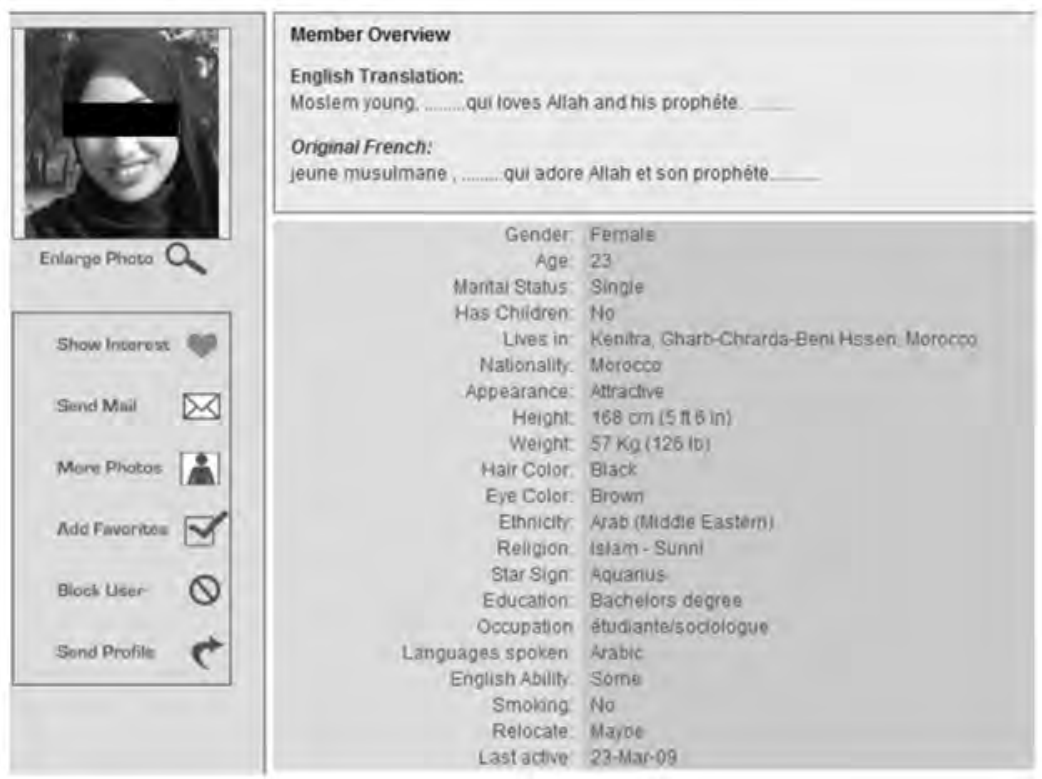


Un seul et même système d'information se tient derrière ces différents sites qui n'en sont dès lors que des façades distinctes. La standardisation n'émerge pas des pratiques, elle est au contraire prescrite d'avance par un principe de collectivisation des moyens : une seule base de données générique est conçue pour tous les sites; la séparation en façades n'est qu'un choix stratégique ${ }^{22}$.

\section{Figure 4. Un profil sur www.mexicancupid.com}

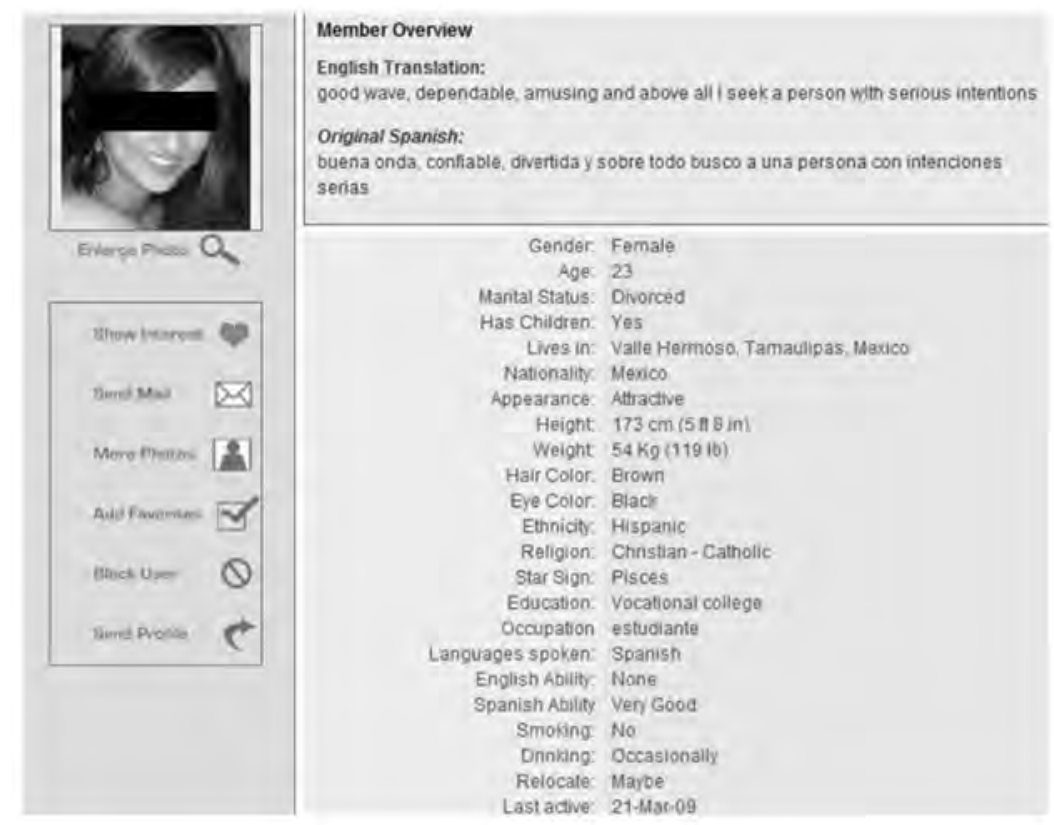

La division par communauté n'est pas seulement un principe d'aiguillage pour les individus qui leur laisserait l'entière décision et responsabilité de leur inscription et de leur participation à une communauté plutôt qu'à une autre ; c'est un principe de distribution et de gestion des individus assignant à chacun d'entre eux selon les caractéristiques de son profil l'espace communautaire qui maximisera ses chances de rencontre. C'est ainsi, pour prendre un exemple, que nous étant inscrit sur le site www.muslima.com, nous avons reçu le lendemain le message électronique suivant, sans que nous puissions

22. Le système est conçu de telle manière qu'il serait possible de créer un unique site, donnant accès à tous les contenus de manière indifférenciée, à très peu de frais et en très peu de temps. Mais cela serait contraire à la stratégie (actuelle) de Cupid Media. 
exactement en déterminer la raison ${ }^{23}$ : «Merci de vous être inscrit à Muslima.com, mais pour votre propre bénéfice et pour maximiser votre nombre de réponses, nous voudrions vous recommander de vous inscrire sur un autre site appelé InternationalCupid.com qui offre les mêmes caractéristiques et avantages ${ }^{24}$. » Précisons que ce message était accompagné d'une exclusion du site, notre identifiant étant devenu inopérant.

Enfin, la standardisation, c'est aussi celle des modalités, du processus, des étapes de la rencontre. Car ce que propose Cupid Media, ce sont des sites dédiés explicitement au dating. Or le dating renvoie à une pratique culturellement située (aux États-Unis d'abord puis dans le monde anglo-saxon) de la rencontre. C'est dans un même mouvement que Cupid Media offre, d'une part, aux différentes communautés des plates-formes qui leur sont spécifiques et, d'autre part, prescrit des modes de relation uniformes. La question serait à présent de savoir dans quelle mesure les individus apprennent à détourner, à jouer avec les possibilités que leur offrent les plates-formes de rencontre pour les adapter à des besoins spécifiques.

\section{Shaadi : la discrétisation des profils au service de la reproduction des communautés indiennes}

C'est un usage de l'ethnicité tout à fait différent que l'on peut observer dans le champ du mariage indien (en Inde et dans la diaspora indienne). En effet, si les sites de rencontre communautaire développés par Cupid Media, bien que fondés sur les différences ethniques/nationales, n'en révèlent pas moins une standardisation gommant les particularités, les besoins et les désirs propres aux différents groupes, le web matrimonial indien, et en particulier le site Shaadi, propose une discrétisation extrêmement fine des profils calquée sur les spécificités de la société et de la culture indienne ${ }^{25}$. C'est ce que nous allons à présent tâcher de démontrer.

« Le commerce du mariage » est une vieille affaire dans la société indienne et ses diasporas. Le sous-continent indien et ses diasporas ont connu dans les

23. Que nous nous soyons inscrits en tant que «chrétien-catholique » ne semble pas être un critère suffisant, puisque le site compte des chrétiens (catholiques ou autres) : 1,48\% des profils que nous avons analysés.

24. Traduit de l'anglais.

25. On ne s'étonnera pas dès lors que Cupid Media n’ait pas développé de sites dédiés à la communauté indienne (bien qu’on retrouve des Indiens musulmans sur Muslima). 
années 1980 « les foires de rencontres » (Pache-Huber, 2005). Les agences matrimoniales ont néanmoins continué de prospérer. L'existence des castes implique des célébrations différentes, d'où la spécialisation des agences sur un ou plusieurs groupes ethniques, religieux et régionaux. Ces agences proposent des " plans de mariages », des tarifs et forfaits divers. Elles ne vendent pas que des profils aux prétendants, elles font de la médiation et, sur demande, s'impliquent dans l'organisation de la rencontre. On retrouve ces agences matrimoniales et leur organisation dans les pays de l'immigration indienne, notamment aux États-Unis, en Grande-Bretagne, au Canada et en Australie. D'autre part, la presse papier spécialisée dans les annonces matrimoniales a accéléré la création de médias consacrés au mariage : les brides magazines. Des annonces matrimoniales trouvaient aussi une place dans les médias généralistes indiens et au-delà, du Sud-Est asiatique dans son ensemble. L'Inde a joué un rôle de propagateur. Son cinéma, qui met la famille au cœur de ses fictions, a insufflé un véritable imaginaire et une culture de "management matrimonial » : parents de prétendants, prétendants, oracles et organisateurs participent pleinement au mariage. Difficile et coûteux en raison des énergies que demande son orchestration, il a une dimension rituelle, cultuelle, initiatique et spectaculaire.

Trois dynamiques méritent d'être relevées lorsque l'on s'intéresse à l'avènement d'internet dans l'univers du mariage indien : il a permis un transfert des traditions dans l'espace numérique, internet devenant un lieu alternatif pour le mariage indien, sans pour autant se substituer aux agences matrimoniales ; il a permis d'agréger tous les acteurs du mariage sur des plates-formes communes ; enfin, il a considérablement élargi le champ des possibles à travers la constitution de larges bases de données de profils.

Le premier site internet de mariage indien est créé en 1990 : BharatMatrimony. Son fondateur, Murugavel Janakiraman s'est lui-même marié grâce à son site internet. Depuis, des centaines, voire des milliers de sites professionnels dédiés au mariage ont vu le jour ${ }^{26}$. Dans le commerce des sites

26. Les sites à fort trafic sont : BharatMatrimony, Starmatrimonials, Muslim matrimonial, Desidesh, Shaadikaro, Free Indian Matrimonial Classifieds, eMatrimonials, Zawaj, Matricorp, iMilap, MyMatrimonial, MarriageSolutions, MatrimonialBank.com, Brahmins Matrimony, Thirumanam, 101 Hot Singles, Asian Matches, Gujarati Matrimonial website, IndiMela Matrimonial, Kamaconnection.com, Pyar.org, Shubhphere.com, Tamilmatrimony, Islamic Singles, GodBless Matrimonials, India Canada Marriage, Kerala Connect et Manamalai. 
internet matrimoniaux, la diaspora indienne est très active en tant que propriétaire mais aussi en tant que cliente. Les Indiens américains et les Indiens britanniques en sont à l'origine. Créé en 1997, le site Shaadi ${ }^{27}$ est le produit d'une double culture : son fondateur, Anupam Mittal, est un ancien étudiant de la Boston University, issu d'une famille de marchands de textiles et d'hommes d'affaires ancrés dans la culture et la société indienne. Ses études de management et son long séjour aux États-Unis n’ont pas bouleversé ses attaches indiennes. Bien au contraire, Anupam Mittal se présente comme le « serviteur » de la société indienne ${ }^{28}$. Son objectif est de rendre « technologiques » et payantes les composantes du mariage tout en respectant la société.

L’originalité de Shaadi est de fusionner toutes les spécificités ethniques, religieuses et de caste de ses prédécesseurs. Il crée une nouvelle valeur ajoutée en proposant une plate-forme matrimoniale à toutes les communautés, les religions et les castes (supérieures) d’Inde. Son originalité réside dans la haute précision des profils des membres et de leurs attentes. Un « profil Shaadi » comprend 40 critères (alors qu'il n'en compte qu'une dizaine sur les autres sites), démultipliant les combinaisons possibles. Le « match » sur Shaadi est très précis. Les recherches peuvent se faire selon le critère professionnel, résidentiel, religieux, racial, par le régime alimentaire, etc. Comme dans un mariage " arrangé », il n’y a pas de surprise pour les prétendants, tandis que les autres sites sont souvent qualifiés de sites de rencontres donc laissant la place à l'incertitude, l'improbable, etc. Shaadi est la dernière étape avant la rencontre des familles. La puissance organisationnelle, fonctionnelle et technologique fait de Shaadi l'un des plus importants sites ${ }^{29}$ internet de mariage indien en Inde et dans sa diaspora d'Amérique, d'Europe et du Moyen-Orient.

27. http://www.shaadi.com.

28. Ce sont des « success stories » des sites matrimoniaux eux-mêmes qui nous sont ici livrées : un travail devrait être mené pour confronter ces « histoires » à la genèse « réelle » de ces platesformes matrimoniales.

29. Il n’y a guère que www.bharatmatrimony.com qui puisse lui être comparé du point de vue de l'audience. 
Figure 5. Profil Shaadi - 40 champs de description de soi et 20 champs de description du (de la) partenaire désiré(e)

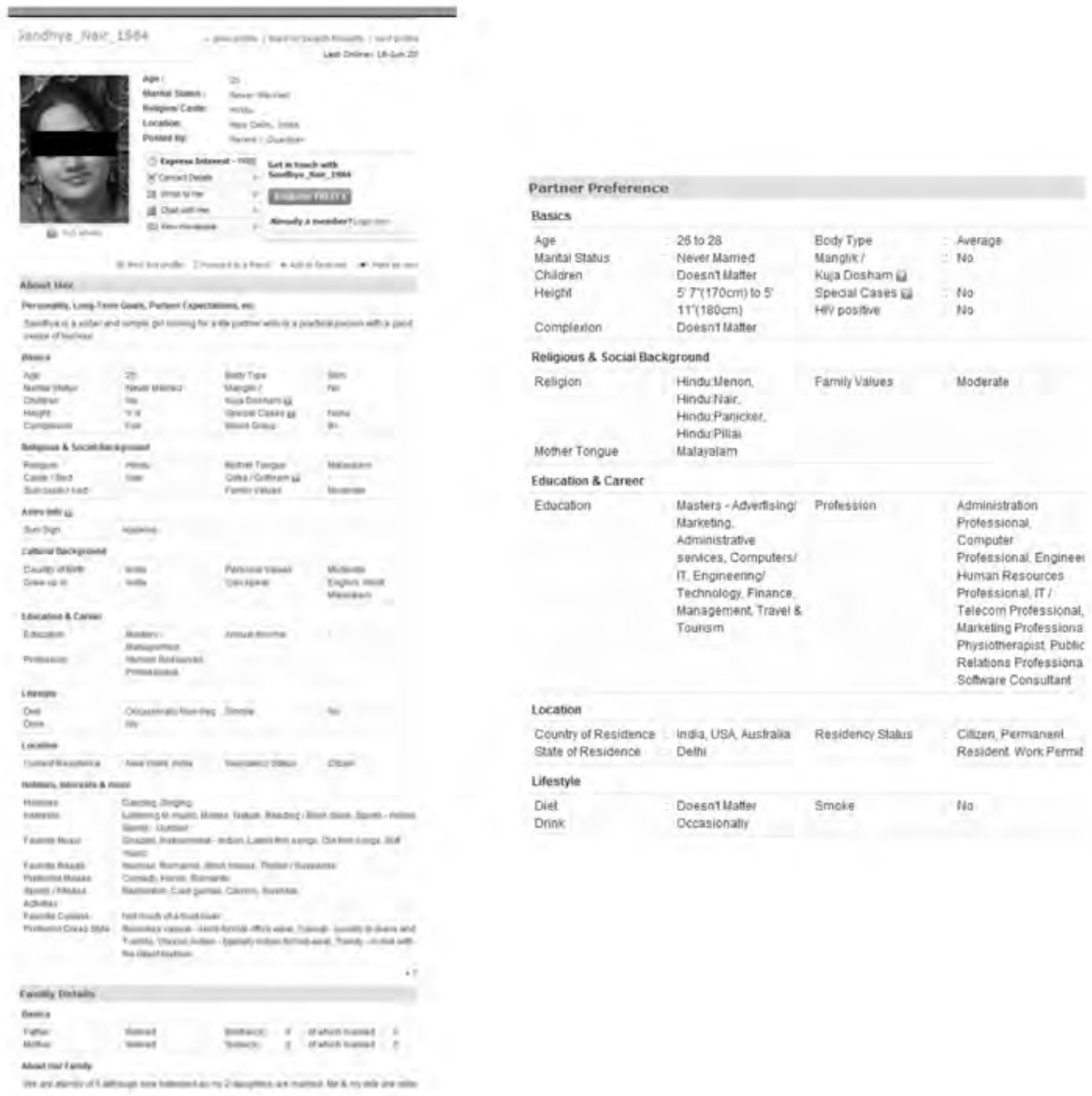

La constitution d'un profil sur Shaadi, à travers lequel l'individu se présente, décrit son partenaire idéal et parfois expose les motivations de sa recherche, dévoile des stratégies diverses. Sur le web, pour être éligible au mariage, il faut des qualités professionnelles, humaines, de caste et même « cosmiques ». Tous les éléments sont importants parce qu'ils sont « codés ». Nos entretiens avec les utilisateurs ont révélé que les « non-dits », les " imprécisions », les « rétentions d'information » et les « omissions » volontaires sont des signifiants matrimoniaux dont les seuls Indiens maîtrisent l'exégèse. Les champs vides ne doivent pas être interprétés comme des données manquantes. Elles 
peuvent traduire une faiblesse, un handicap social ou astrologique. La formule doesn't matter peut signifier une certaine ouverture sur une question précise et un conservatisme sur d'autres. La clause Family Values peut servir à délimiter les champs de l'ouverture sur la conception du monde et l'ouverture à une certaine occidentalisation. Cette signalétique des « conjoints potentiels » assure une circulation des codes et valeurs, d'alliance entre les familles ${ }^{30}$. Les Indiens exigent, pour le mariage, des conditions sociales, économiques, politiques, religieuses et astrologiques similaires (Banerjee, Duflo, Ghatak, Lafortune, 2009) ${ }^{31}$. Shaadi véhicule une culture « indo-centrée » et participe à la construction d'une société qui, à l'échelle globale, est de plus en plus « castée », de plus en plus « ethnicisée ».

Enfin, ce que nous dévoile Shaadi, c'est que l'économie du profilage peut s'associer à une économie de services " traditionnels " (à différencier des services de «matching », de communication, etc.). Ainsi, People Group, propriétaire de Shaadi, propose une gamme de services (intégration verticale) préparant, accompagnant ou succédant à la rencontre sur Shaadi. C'est tout d'abord ShaadiTimes ${ }^{32}$, journal qui donne des informations et des conseils juridiques. Les mariés y trouvent des liens et des adresses pour préparer leur mariage, leur voyage de noces, etc. Des sollicitors vendent aux shaadinautes des conseils juridiques pour émigrer et leur présentent les lois sur le mariage dans les pays de migration. Vient ensuite Astrolife ${ }^{33}$ qui propose des services d'astrologie permettant notamment de déterminer la concordance des astres des mariés : le manglik. Astrolife fournit des horoscopes quotidiens, des chartes de naissance (birth chart), des « validateurs » de couple (match making), etc. Enfin, Shaadi a mis en place un dispositif complémentaire, les ShaadiPoints, authentiques centres de conseil, mettant à disposition des bases de

30. Ceci est néanmoins susceptible de témoigner d'une certaine limite à la discrétisation des profils dans la mesure où, même pour un(e) Indien(ne), ces champs pourraient être vecteurs d'une incertitude. Seule une étude des usages en situation permettrait d'avancer des conclusions sur ce point.

31. Ces auteurs ont montré que la caste est encore en Inde un critère prépondérant de la recherche d'un conjoint alors même que le développement économique tend à affaiblir la corrélation entre caste et revenu. Si les mariages hors-caste deviennent plus fréquents, ceux-ci sont très souvent des « mariages d'amour » (à la différence des " mariages arrangés »). Les auteurs concluent que «l'institution que les forces économiques ne sont pas capables de détruire peut être mise en danger par l'amour ». Puisque Shaadi favorise et renforce le mariage arrangé, on peut émettre cette hypothèse qu'il contribue à renforcer l'institution du mariage intra-caste.

32. http://www.shaaditimes.com/

33. http://www.astrolife.com/ 
données pour rechercher le partenaire idéal, arranger au mieux le mariage. Shaadi se donne ainsi pour objectif de contrôler toutes les fonctions stratégiques du mariage. L'économie du profilage est, en un sens différent de celui employé jusqu'ici, une économie de l'au-delà du profil.

\section{Femmes de l'Est par correspondance - la vente d'adresses}

Enfin, une économie du profilage ethnique est également à l'œuvre dans le champ des rencontres internationales, dans lequel rencontre signifie mobilité, ou plus précisément migration des femmes de l'Est/Sud vers l'Ouest/Nord où réside le potentiel partenaire dans le cadre d'un projet qui devient exclusivement projet de mariage et qui trouve dans l'inégalité économique des partenaires l'un de ses fondements Nous considérerons ici à titre d'exemple les unions franco-« pays de l'Est » médiées par Internet (marché lucratif dans lequel les escroqueries sont nombreuses).

La majorité des agences matrimoniales ayant investi ce marché sont exclusivement présentes sur le web ${ }^{34}$. Nous sommes là en présence d'une authentique économie du mariage qui se fonde sur la vente d'adresses électroniques et donc d'un accès à des femmes/candidates. Ce phénomène n'est pas nouveau; il n'est pas né avec Internet ainsi que nous le rappelle Rolando B. Tolentino (1996) ; il renvoie à ce qu'on désigne en anglais du nom de mail-order brides (épouses par correspondance). Il n'en reste pas moins que le web s'est révélé être un vecteur privilégié grâce à la constitution de vastes bases de données de profils (les « fichiers de femmes »), catalogues accessibles à travers des interfaces de recherche multicritères. Nous pouvons à nouveau parler d'une économie du profilage.

34. Nous pouvons proposer une liste, extrêmement loin d'être exhaustive, d'agences dédiant leurs services à des hommes français : Inter-mariage.com, Annabarmina, Europalove, Activelovemeeting, Premium-mariage-international, Accords-franco-russes, Natclub. Si certaines d'entre elles ne cherchent pas à masquer ce qui constitue la source première de leur activité, à savoir la vente d'adresses («La première étape consiste à nous acheter les informations concernant l'élue de votre cour, ensuite vous pouvez leur envoyer une lettre et commence la grande aventure »), d'autres entendent marquer leurs différences à l'égard, non seulement des " agences matrimoniales classiques ", mais aussi des "clubs de rencontre vendant des adresses de femmes russes ». Il semble néanmoins que ces différences ne soient que des différences déclarées, les services proposés d'un site à l'autre étant à peu près identiques. L'enjeu est notamment d'échapper à la méfiance de potentiels clients à l'égard du « SCAM », terme désignant l'escroquerie sur Internet ; c'est ainsi que des sites tels www.anti-scam.org proposent des catalogues «blacklist » de profils (nom(s), photos, ville, date de naissance, coordonnées, etc.) associés à des pratiques frauduleuses. 
La logique économique dans l'univers du mariage franco-« pays de l'Est » est centrale. Nous sommes là en présence d'un «marché de l'amour» dans lequel la valeur monétaire des services proposés est toujours explicitement exposée ; les sites concurrents ne se distinguent guère par l'originalité de leur offre de services qui dévoile une véritable rationalisation de l'exposition de soi, ou plus exactement, une quantification en vertu de laquelle à chaque degré de dévoilement peut être associée une valeur monétaire :

- Ils proposent aux utilisateurs de devenir membres, ce qui leur permet d'accéder, de manière illimitée ou non, à différents services.

- L’envoi de message, la correspondance électronique : celle-ci répond à une tarification dont le principe est simple : plus grand est le nombre d'adresses achetées (de mises en relation demandées), moins le coût d'une adresse à l'unité est élevé. C'est une économie d'échelle. Le coût des messages dépend également de leur volume : attacher des photographies peut requérir un investissement supplémentaire.

- La traduction : ce service accompagne le précédent pour des raisons évidentes de compréhension. Si le tarif est fonction du nombre de mots à traduire, il peut aussi dépendre de la nature du message : traduire de la poésie est plus coûteux que traduire de la prose !

À côté de ces agences online, on en trouve d'autres, beaucoup moins nombreuses, dont l'activité est à la fois online et offline et qui s'identifient plutôt aux agences matrimoniales traditionnelles. C'est le cas notamment de Eurochallenges ${ }^{35}$, agence matrimoniale française créée en 1996 ; ses débuts, alors qu'elle ne se consacrait encore qu'au mariage franco-français, furent laborieux ; les difficultés financières ne tardèrent pas à faire leur apparition. C'est alors qu'elle s'est spécialisée avec succès dans le domaine du mariage international. Le succès commercial d'Eurochallenges ne fait pas de doute : Anne Muser, la fondatrice de l'agence prétend que celle-ci a participé à la création d'environ 10000 unions stables depuis sa fondation en $1996{ }^{36}$.

Eurochallenges expose sur son site un catalogue de profils de prétendantes étrangères, les catégories étant : " femmes russes et d'Europe de l’Est », "femmes

35. http://www.eurochallenges.com.

36. http://www.eurochallenges.com/526_mariage-femme-de-1-est.html. Dans cette même interview, Anne Muser présente des réussites particulières, des « success stories ». C’est un procédé très commun dans les sites matrimoniaux. Il faudrait ajouter qu'encore une fois, c'est l'histoire même de l'agence qui est présenté comme une « success story ». 
asiatiques », « femmes africaines, d’Orient et d'Océan Indien ». Les « femmes de l'Est » représentent près de $70 \%$ du nombre total de profils. La recherche dans le fichier de femmes et la consultation de profils détaillés constituent une introduction libérée de tout engagement. Une telle exposition du profil de l'autre (potentiel « objet d'amour »), de son « identité » et de son corps, doit favoriser un " passage à l'acte ${ }^{37}$. Ici débute le travail de l'agence matrimoniale traditionnelle. Eurochallenges est liée à un ensemble d'agences matrimoniales partenaires dans les pays d'origine des femmes (la majorité étant en Russie) ; ces agences sont sélectionnées sur le critère de leur aptitude à travailler elles-mêmes en collaboration de telle manière que se bâtisse un véritable réseau du mariage international fondé sur le partage et la circulation des profils, réseau qui est certes piloté par l'Ouest (en l'occurrence la France) mais dans lequel les pays d'origine jouent également un rôle décisif.

Concluons ici en soulignant que la niche économique qu'exploitent les acteurs du mariage mixte est étroitement dépendante de l'ethnicisation en vertu de laquelle est produite la catégorie "femme de l'Est ». Cette catégorie n'informe pas seulement le discours de ces acteurs (en évoquant tout particulièrement les idéaux de la famille et la féminité incarnée par la « femme de l’Est ») mais aussi la structure même des sites web, qui repose sur la catégorisation des femmes et sur des critères de recherche selon le «type ethnique » ou la nationalité, etc. Même des agences prétendument plus « sérieuses » telles qu'Eurochallenges ne peuvent masquer cette ethnicisation des rapports amoureux : la "Région du monde » est en effet l'un des deux critères de recherche (avec l'âge) proposés immédiatement sur la page d'accueil d'Eurochallenges ${ }^{38}$.

\section{Les usages (économiques) de l'ethnicité}

Les exemples précédents nous ont révélé que si une économie du profilage ethnique est bien à l'œuvre sur l'ensemble des plates-formes du WMM, il ne s'ensuit aucunement qu'il n'y ait qu'une et une seule manière de " faire du commerce » avec l'ethnicité. C'est pourquoi nous aimerions synthétiser les trois usages de l'ethnicité qu'il nous a été donné d'observer.

37. Ce dernier suppose que le client prenne rendez-vous dans l'une des agences «physiques » d'Eurochallenges (au nombre de 14 en France).

38. Le fait que ce dernier critère n'ait plus rien de géographique est prouvé en ce que certaines femmes inscrites et vivant en France sont classées sur le site en fonction de leur « pays d'origine ». 
L'ethnicité se traduit dans l'interface d'Eurochallenges par une " région du monde ", tacitement d'origine ; mais ces origines géographiques sont ici le faux-nez de catégories ethniques. La particularité de ce modèle tient à ce nondit : parce qu'elles ne sont pas explicites, des catégories imaginaires produisent l'ethnicité (typiquement « la femme de l'Est »). Eurochallenges joue de l'ethnicité sur deux plans : une catégorisation qui délimite l'offre en largeur, et la suggestion générale d'un imaginaire géographique, d'un fantasme " exotique » qui spécifie l'offre en qualité. Dans ce modèle d' « union transnationale », l'ethnicité est simplement une qualité de l'offre (et de la marque Eurochallenges).

Figure 6. Extrait du fichier de femmes d'Eurochallenges

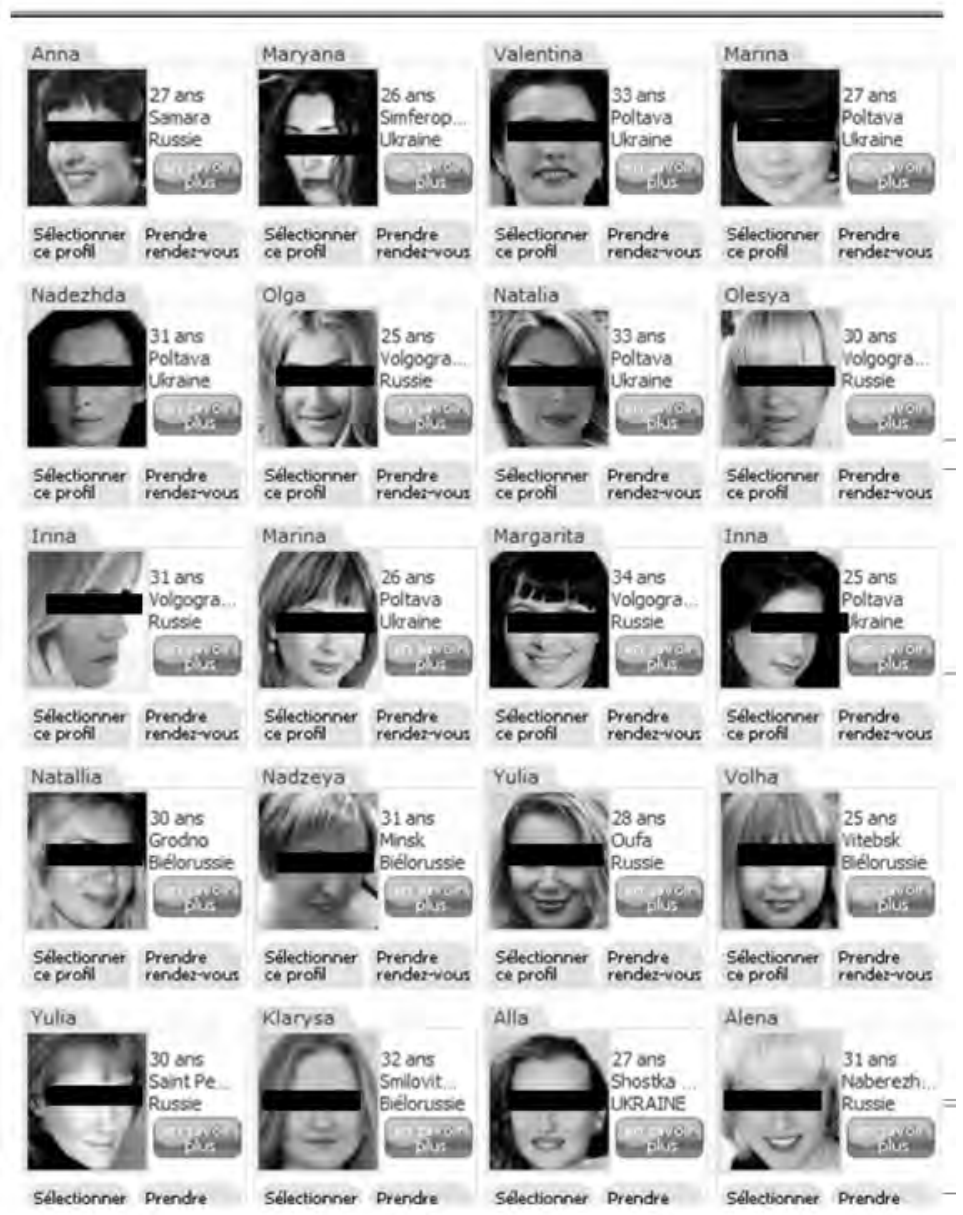


Le modèle Cupid Media est plus complexe, puisque les catégories ethniques y sont séparées d'autorité. C’est même sa particularité : pour déposer son profil, il est nécessaire de s'identifier par une catégorie, religieuse ou nationale, incarnée par un site donné du système Cupid. Ces sites étant imperméables les uns aux autres, le modèle repose sur l'idée que les personnes partagent le projet de s'unir au sein de leur propre catégorie (ethnique). L'ethnicité, dans ce modèle « communautariste », est le critère permettant la segmentation de l'offre.

Shaadi quant à lui accompagne l'ethnicité plus qu'il ne l'instrumentalise. L'ethnicité est prise en compte en tant qu'elle revêt une valeur pour les personnes en recherche d'union. En particulier les multiples critères ayant trait à l'ethnicité peuvent être déclinés dans les profils; la catégorie ethnique n'est pas prescrite (en dehors du fait que le site est dédié aux Indiens) mais au contraire disséminée dans les données. En ce sens, elle s’accorde aux besoins de la communauté, à savoir cerner les appartenances (notamment) ethniques d'une personne. Dans ce modèle « communautaire », l'ethnicité est une propriété des personnes, et valorisée en tant que telle.

L'ethnicité se décline le long de ces trois modèles, d'une propriété de l'offre (Eurochallenges) à une propriété des personnes (Shaadi). On pourrait alors émettre cette hypothèse que, plus tôt l'ethnicité est imposée par le système, moins elle peut être investie/gérée par les personnes pour se décrire. Or comme Shaadi le montre bien, l'ethnicité d'une personne nécessite un décodage dont les clés sont détenues par la communauté. Au contraire, l'exhibition suggestive de l'ethnicité peut déboucher sur un fantasme qui ne fonctionnera qu'au loin des personnes concernées (homme français et femme russe, dans les deux sens).

On n'explicitera néanmoins pleinement l'économie du WMM qu'en comprenant comment à la question des usages de l'ethnicité s'articulent les critères de genre et les enjeux de migration.

\section{GENRE ET MIGRATIONS DANS LE WMM}

\section{Répartition hommes/femmes sur Muslima}

On l'a vu, les sites de la gamme Cupid Media révélaient un effacement des différences entre communautés. Il paraissait ainsi presque impossible de 
trouver des critères de différenciation de ces sites, excepté la désignation du public visé, le nom de la communauté ciblée par chacun d'eux. Cependant, du point de vue des contenus, c'est-à-dire des membres eux-mêmes et de leurs profils, des différences importantes peuvent être identifiées. Nous paraissent à ce titre décisives les multiples configurations de l'ethnicité/nationalité, d'un côté, du genre de l'autre.

Prenons l'exemple du site Muslima. Les données sont basées sur les 9000 profils d'hommes et 9000 profils de femmes extraits. Nous avons tout d'abord représenté la répartition des genres dans chacun des pays (de résidence) dont les membres représentent (soit chez les hommes, soit chez les femmes) plus de $1 \%$ du total des inscrits sur le site. Nous obtenons l'histogramme suivant :

Figure 7. Répartition hommes/femmes selon le pays de résidence sur Muslima

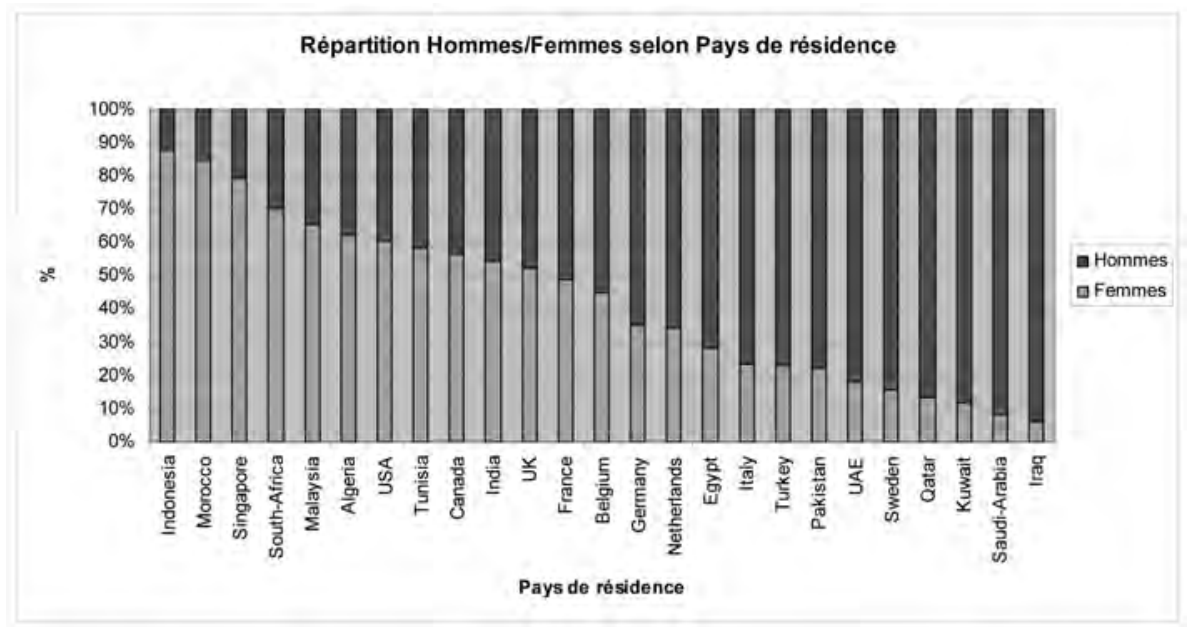


Figure 8. Répartition hommes/femmes selon la nationalité sur Muslima

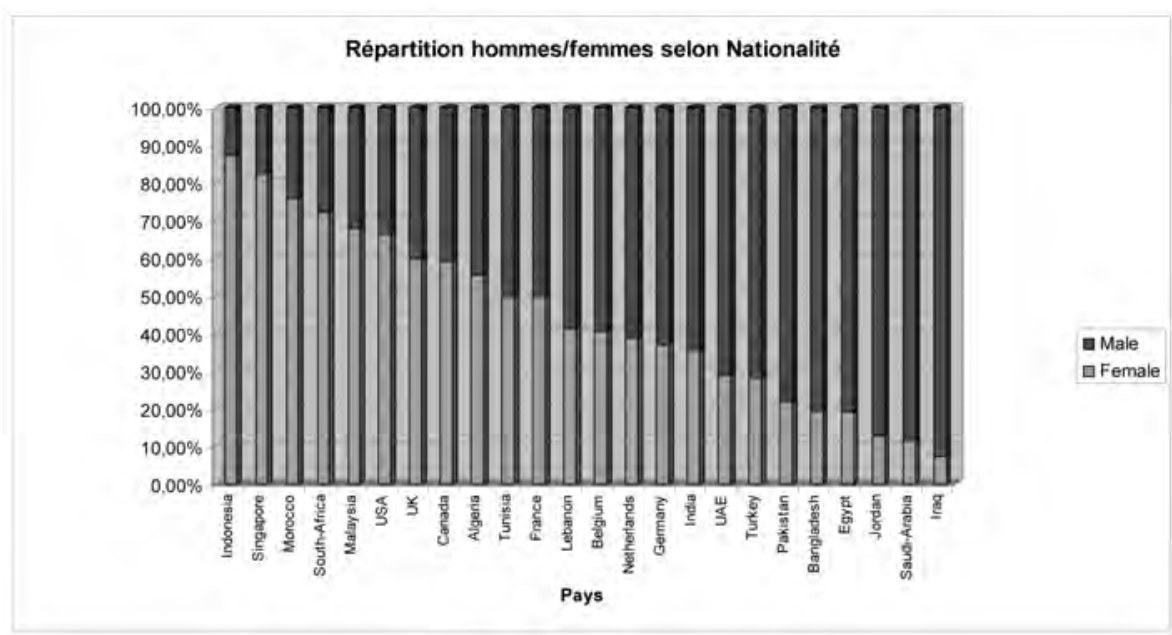

On observe donc de manière schématique (on ne s'occupera pas ici des exceptions) que dans les pays asiatiques (Indonésie, Malaisie, Singapour) ainsi que dans les pays du Maghreb, les femmes sont largement majoritaires; dans les pays d'émigration (Royaume-Uni, France, etc.), la répartition homme/femme est équilibrée. Dans les pays du Moyen-Orient, la proportion d'hommes est beaucoup plus forte que celle des femmes. Des observations analogues peuvent être faites au sujet des relations entre genre et nationalité.

\section{Une distribution internationale du genre}

Mais c'est dans le domaine des rencontres intercommunautaires que cette distribution des genres prend toute sa signification. Cupid Media propose un ensemble de sites dédiés à la rencontre intercommunautaire comme www.internationalcupid.com, www.asianeuro.com, www.russianeuro.com, voire même des sites jouant explicitement avec les fantasmes les plus éculés (mais toujours prégnants) de l'amour interracial : http://www.interracialcupid.com/. Mais loin d'être circonscrit ou de s'ajouter simplement aux sites intra-communautaires, le domaine des rencontres intercommunautaires pénètre ces derniers et autorise une circulation, malgré (et à travers) le cloisonnement communautaire. C'est ainsi que plusieurs sites autorisent la présence d'individus extérieurs à la communauté - incitent à la « transgression » - de telle manière que la différence 
culturelle, ethnique peut devenir critère d'affinités et même assurer le succès d'un site dit communautaire. Cette circulation intercommunautaire n'est pas généralisée ${ }^{39}$; elle suit certaines " trajectoires » et s'exprime à travers une distribution internationale du genre : ce sont presque exclusivement les hommes qui « se déplacent » sur des sites d'une autre communauté que la leur ${ }^{40}$. Les « déplacements » intercommunautaires ne s'opposent pas aux logiques de la communautarisation ; ils se fondent tout autant sur la différence nationale/ ethnique/raciale, sur ce que nous avons appelé profilage ethnique, que les rencontres intracommunautaires (cf. le drapeau national associé à chaque profil sur l'ensemble des sites Cupid Media) ; l'ethnicité n'est en aucun cas devenue indifférente ; à l'homophilie s'est substituée l'hétérophilie. Les dispositifs d'échange (intercommunautaire) de femmes, pour paraphraser Lévi-Strauss, participent par conséquent à la polarisation communautaire et à l'économie qui la nourrit.

Illustrons cela par quelques exemples. Sur le site www.muslima.com, 90,8 \% des femmes et $87,3 \%$ des hommes vivant aux États-Unis, et $88,7 \%$ des femmes et $84,9 \%$ des hommes vivant en France, sont musulmans. Les membres non musulmans (la plupart ayant rempli le champ de religion avec la mention «other » et dans une moindre mesure " no religion ») sont donc peu nombreux. Au contraire, sur le site www.filipinaheart.com, la distribution genrée de l'ethnicité et de la nationalité est éloquente. Aux États-Unis, 89,9\% des femmes sont philippines tandis que $86,2 \%$ des hommes sont américains (seuls $6,9 \%$ sont, du point de vue ethnique, asiatiques). En France, $87 \%$ des femmes sont philippines et 90,4\% des hommes sont français (seuls 5,1\% sont « asiatiques »). Enfin, www.mexicancupid.com est intéressant dans la mesure où si, aux États-Unis, il est investi par 70,7 \% de femmes et 57,7 \% d'hommes « hispaniques » ou " mixed », en France, il l'est par 71,4 \% de femmes mais seulement 20,4\% d'hommes de ces groupes ethniques. Ce sont bien sûr les réalités migratoires - la forte immigration mexicaine aux ÉtatsUnis - qui entrent ici en ligne de compte.

39. Que cette circulation reste minoritaire est peut-être même une condition pour que continue à s'exercer la logique de l'appartenance à l'œuvre dans ces sites communautaire. Elle n'en est pas moins paradigmatique.

40. Ce n'est pas nécessairement dire que les femmes soient plus « communautaires » que les hommes - ainsi que ce pouvait être le cas sur les sites de rencontre généralistes. Mais seulement que dans ce jeu - qui se joue à deux - de la rencontre intercommunautaire, c'est l'homme qui « sort» de sa communauté pour aller chercher une femme qu'il reconduira peut-être après auprès de cette communauté - induisant une mobilité (géographique) de la femme. 
Figure 9. Répartition des nationalités selon le genre sur Filipinaheart (pays de résidence $=$ France)

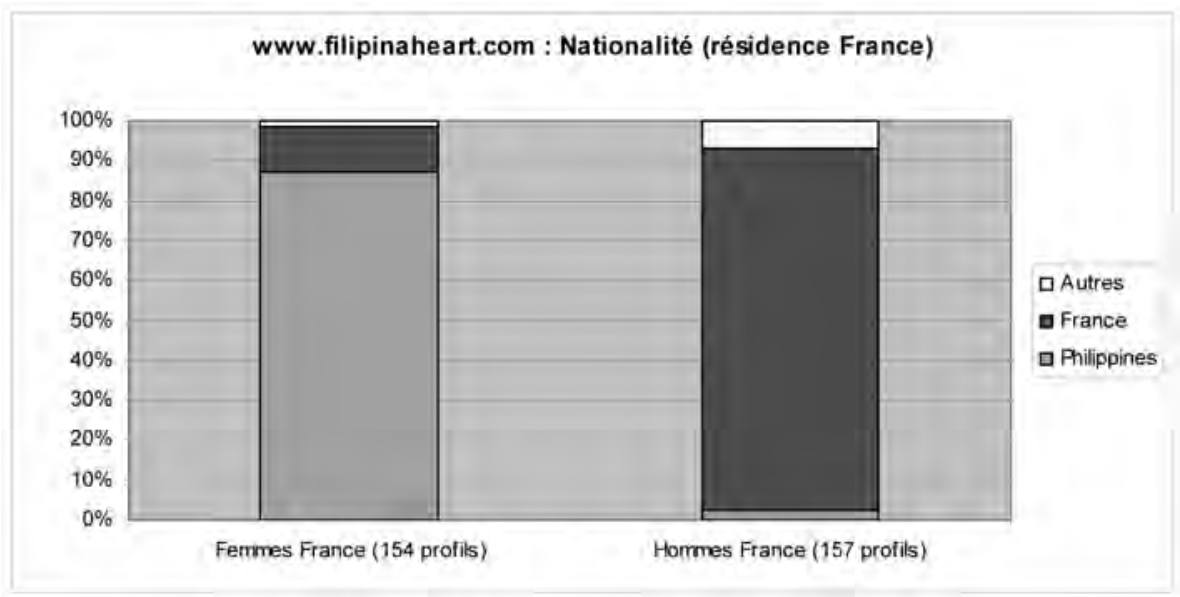

Figure 10. Répartition des nationalités selon le genre sur Filipinaheart (pays de résidence $=$ États-Unis)

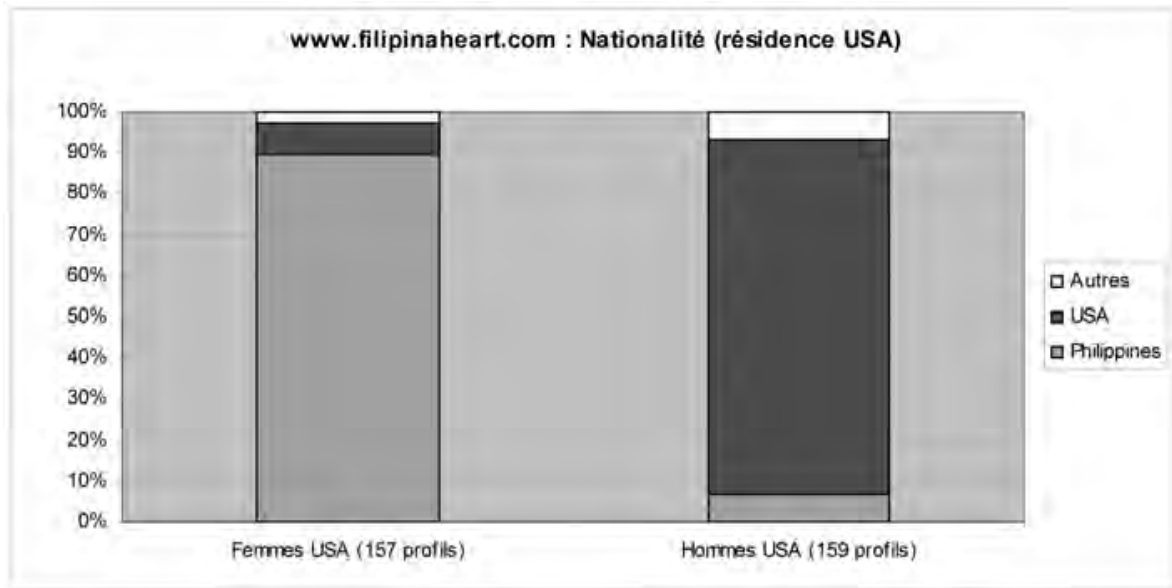

Nous avons poursuivi nos investigations en créant un profil de femme sur le site Mexicancupid : une femme mexicaine de 23 ans, vivant à Puebla au Mexique, célibataire sans enfants, et laissant ouvert le champ « voulant déménager dans un pays étranger ${ }^{41}$ (réponse : « maybe »). Nous désirions ainsi connaître

41. Nous reviendrons plus loin sur la présence de ce champ du profil. 
le profil des hommes - nationalité, ethnicité, pays de résidence, âge, etc. intéressés par notre profil fictif ${ }^{42}$. Par souci déontologique, nous nous sommes interdits de poursuivre les échanges et nous sommes limités aux profils ainsi qu'aux premiers messages électroniques envoyés par les prétendants. Nous avons ainsi pu voir que parmi les hommes ayant témoigné de leur intérêt (171 « preuves d'intérêt ») avec ou sans message associé), près de 70 \% étaient de nationalité mexicaine, tandis qu'un peu plus de $20 \%$ étaient de nationalité américaine (en ce qui concerne l'ethnicité, près de $25 \%$ se déclarent « Caucasian (White)). Du point de vue du pays de résidence, $63 \%$ vivent au Mexique et $33 \%$ aux États-Unis. Ceci indique, et les messages par ailleurs reçus le prouvent, que nombreux sont les hommes mexicains recherchant via ce site, une partenaire mexicaine vivant au Mexique. Il y a également une corrélation forte entre la nationalité et l'âge du prétendant, ainsi que le montre le graphique suivant :

Figure 11. Âge des prétendants selon la nationalité sur MexicanCupid

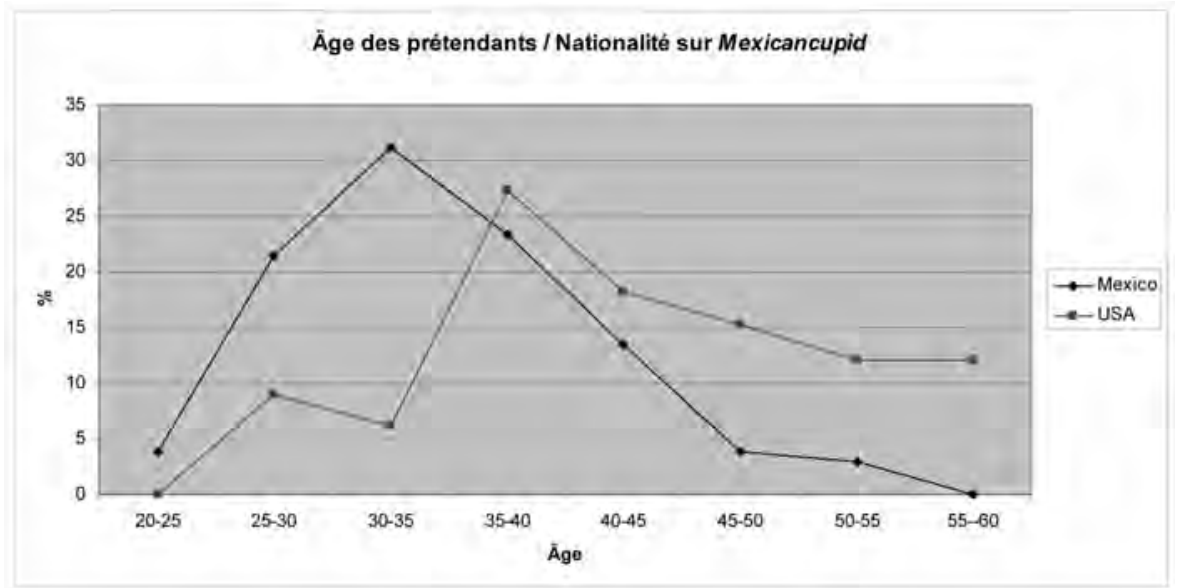

\section{«Désir de migration»}

Le site MexicanCupid est donc un lieu où se déroulent des phénomènes hétérogènes : rencontre intracommunautaire (intranationale) dans le cadre des frontières du pays, rencontre intracommunautaire impliquant le pays d'origine et les pays d'accueil (voire les seuls pays d'émigration), rencontre intercommunautaire impliquant là aussi de potentielles migrations. L’idée de déplacement

42. L'expérimentation a duré trois semaines, du 8 mai au 29 mai 2009. 
physique est au cœur même des échanges sur le site ainsi qu'en témoignent les messages que nous avons reçus ${ }^{43}:$ « Je veux venir vous rencontrer au Mexique si vous êtes intéressée. Je peux déménager au Mexique ou vous parrainer pour venir aux États-Unis une fois que nous nous connaîtrons bien ${ }^{44}$; ou encore, ce message d'un Espagnol : «Un de mes meilleurs amis va vivre à Puebla en juillet ; ainsi la visite est obligatoire et j'aimerais connaître des gens de là-bas et si elles sont si belles que toi, c'est beaucoup mieux ${ }^{45}$; un dernier message d'un homme vivant à Mexico : "Je sais que vous pourriez répondre que la distance est un problème (...) mais pour l'amour il n’y a pas de limites. »

L'enjeu de la migration, des déplacements géographiques, est ainsi décisif dans la gamme des sites Cupid Media : en témoigne le fait que, sur l'ensemble des sites, le critère « désirant déménager dans un pays étranger » (avec pour réponses possibles : « yes », « no » ou « maybe ») doit être renseigné lors de l’inscription. Ceci vaut par exemple pour Muslima. Les histogrammes suivants montrent, pour chaque pays de résidence, le pourcentage d'hommes et de femmes ayant répondu « oui » à la question « désirant déménager dans un pays étranger » :

Figure 12. Désir de migration selon le pays de résidence sur Muslima (genre $=$ femme)

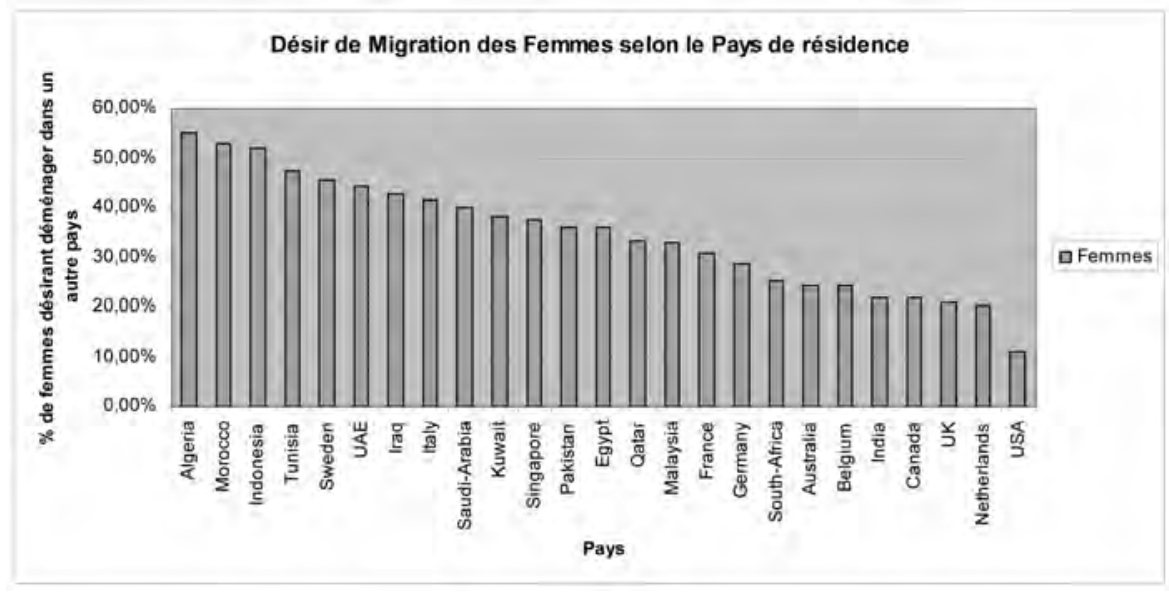

43. Ces messages témoignent également d'une volonté (de la part des hommes du moins) de sortir le plus rapidement possible du cadre du profil : c'est ainsi que l'adresse mail personnelle est presque toujours renseignée dès le premier message envoyé sur le site. Très souvent, est également donné (ou demandé) le contact msn messenger. Enfin, nombreux sont les prétendants à demander l'envoi de photographies.

44. Traduit de l'anglais.

45. Traduit de l'espagnol. 
Figure 13. Désir de migration selon le pays de résidence sur Muslima (genre $=$ homme)

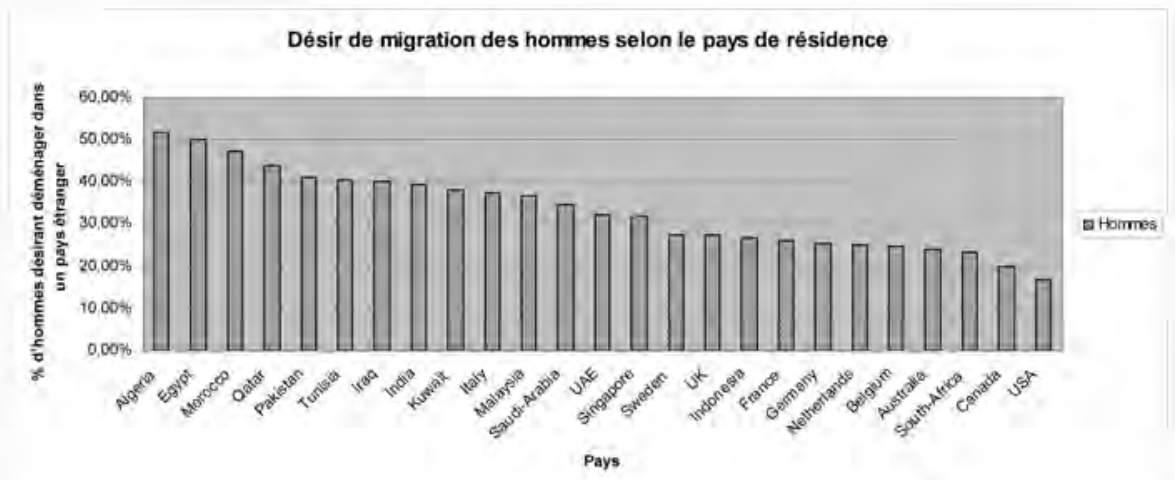

Dans les pays du Maghreb ainsi qu'en Indonésie ${ }^{46}$, le pourcentage de femmes désirant migrer est très fort (de $47 \%$ à $55 \%$ ). Des observations analogues peuvent être faites pour les hommes, à ceci près qu'il faut ajouter l'Égypte, le Qatar ou encore le Pakistan. Au contraire, ce pourcentage est presque toujours très faible dans les pays d'émigration. On comprend par conséquent que Muslima peut être conçu comme un instrument privilégié dans un projet migratoire.

\section{Rencontre internationale et migration}

Le triplet nationalité-genre-mobilité que nous avons tâché jusqu'ici d'illustrer ne se présente nulle part sous une forme plus « épurée » que dans le domaine spécifiquement dédiée à la rencontre internationale. Nous en revenons par conséquent au domaine des unions " Ouest-Est » mais en considérant tout d’abord un site développé par Cupid Media : RussianEuro.

Nous avons créé un profil de femme : 24 ans, divorcée sans enfants, ne précisant pas si elle désire déménager à l'étranger ou non (réponse " maybe ») ${ }^{47}$. En droit, nous aurions pu être contactés tant par des hommes de nationalité russe ou plus généralement des pays de l'Est que par des hommes provenant

46. Ce qui témoigne d'une corrélation entre genre et « désir de migration » puisque ces pays étaient ceux dans lesquels la proportion de femmes était largement supérieure à celle des hommes.

47. L'expérimentation a duré trois semaines, du 8 mai au 29 mai 2009. 
d'autres régions du monde (avant tout Europe et États-Unis). Il n’en fut rien. Les six nationalités les plus représentées parmi les membres du site ayant témoigné de leur intérêt pour notre profil (227 «preuves d'intérêt ») sont les suivantes : états-unienne (16,4\%), française (13,6 \%), italienne (9,6 \%), néerlandaise (7,9\%), allemande (5,65 \%), anglaise (5,65\%), turque $(3,95 \%)^{48}$. On a pu par ailleurs voir que l'âge moyen des « prétendants » était très largement supérieur à l'âge de notre profil fictif.

RussianEuro est bien un site dédié exclusivement ou presque aux rencontres internationales et dans lequel la relation entre distribution des nationalités et distribution des genres suit une loi très simple : à quelques exceptions près, on ne retrouve pour chaque nationalité, soit que des hommes, soit que des femmes. Il n'est guère étonnant par ailleurs que la question des déplacements soit présente dès les premiers échanges de messages : «À propos de la femme que je veux : je veux qu'elle veuille vivre en Égypte. "; "J'ai vu ton profil ; mais tu as la possibilité de venir en vacances en Italie pour faire connaissance? "; " Je ne suis pas encore allé en Russie. À quoi ça ressemble où tu vis ?"; " Je suis professeur à l'université de Bari mais voyage souvent autour du monde pour des raisons scientifiques. "; "Connais-tu Istanbul et la mer Méditerranée ? Y es-tu déjà allée ? " ${ }^{49}$

Mais si la mobilité, le déplacement sont au cœur de l'économie du mariage international, ce n'est pas seulement au sens où ce mariage impliquera migration. La mobilité est en jeu dès le moment du choix, de la sélection du partenaire. En effet, notre étude du champ du mariage franco-« pays de l'Est» nous a dévoilé que le voyage romantique était au cœur même de l'économie du profilage en tant que celle-ci implique la sortie du profil. Le « voyage romantique » désigne le déplacement du prétendant en Russie (ou un autre pays de l'Est) pour rencontrer sa, mais plus généralement ses potentielles futures épouses ${ }^{50}$. C’est la concrétisation de la transaction débutée avec la vente d'adresses. Les agences peuvent s'occuper d'une partie ou de l'intégralité de l'organisation de ces voyages : invitation (nécessaire pour l'obtention d'un visa russe par exemple), réservation d'hôtel ou d'appartement, contacts sur place (avec souvent une « conseillère » dédiée), organisation des rendez-vous, etc. En réalité, bien plus qu’un service se « greffant » sur le web matrimonial,

48. Les Turcs représentent $20 \%$ des membres nous ayant écrit un message.

49. Tous ces messages ont été traduits de l’anglais.

50. C'est justement l'avantage du « voyage romantique » que de permettre des rendez-vous multiples. Dans le cas où l'homme voudrait rencontrer une et une seule femme, il a la possibilité de la faire venir dans son pays. 
le voyage romantique constitue d'une certaine manière son but. Nulle part plus qu'ici en effet les acteurs du web matrimonial disposent des moyens du dépassement du profil en vue de la rencontre physique. Le déplacement d'un côté, le profilage de l'autre, constituent les deux faces d'une même pièce.

\section{Shaadi : une migration choisie}

Le web matrimonial indien présente lui aussi une articulation intéressante de l'ethnicité, du genre et de la mobilité. Shaadi est en effet un site qui s'adresse aux castes et classes aisées. Ainsi, il favorise avant tout la mobilité des groupes et professions les plus recherchés en Europe et dans le monde : informaticiens, médecins, dentistes, infirmières, etc. Il est un vecteur de la circulation des élites. Il est le lieu d'une politique informelle de migrations choisies, autorégulées, spontanées, qui ignore les corridors officiels tout en demeurant dans une logique du choix ${ }^{51}$.

Ne croyons pas cependant qu'un site tel que Shaadi ne soit qu'un « facilitateur » de migrations. Les données suivantes sont établies sur la base de l'extraction de 4500 profils de femmes et 4500 profils d'hommes. Aux États-Unis, $80,2 \%$ des femmes recherchent prioritairement un conjoint dans ce même pays et 6,9\% en Inde (12\% de Doesn't matter). En Angleterre, elles sont $79,2 \%$ à rechercher sur place et seulement $2,2 \%$ en Inde $(10,9 \%$ de Doesn't matter). En Inde enfin, les données sont légèrement différentes dans la mesure où elles ne sont que $55,5 \%$ à rechercher un partenaire en Inde, ce qui ne signifie cependant pas qu'elles privilégient un pays en particulier puisqu'elles sont 38,8 \% à indiquer Doesn't matter. Les chiffres ne sont pas fondamentalement différents chez les hommes. On observe néanmoins une plus forte tendance pour les émigrés indiens à rechercher une femme dans le pays d'origine : ils sont 20,4\% aux États-Unis et 10,4\% en Angleterre à rechercher une conjointe en Inde. Shaadi révèle, plus qu'il ne rend possible, la migration ( « choisie » au sens où les critères du mariage arrangé restent essentiels) des femmes indiennes vers les pays d'émigration. Nos données nous permettent par ailleurs d'observer un autre phénomène, à savoir l'attrait qu'exercent les

51. Il serait intéressant d'étudier si cette thèse ne vaut pas pour le WMM en général. Elle bousculerait évidemment nombre d'idées reçues, à savoir : premièrement, que l' « immigration choisie » est une politique mise en œuvre exclusivement par les instances étatiques, une politique allant à l'encontre des pratiques migratoires non régulées, conçues a priori comme entrant en dissonance avec les critères de sélection imposés par le régulateur ; deuxièmement, que le mariage, assimilé idéologiquement au « regroupement familial », attire et provoque le déplacement d'une population non désirée dans les pays de destination. 
États-Unis, puisque, chez les femmes, les États-Unis représentent plus de $5 \%$ des premiers choix du pays de résidence souhaité du conjoint dans $81,3 \%$ des 32 pays de résidence étudiés (par comparaison, pour le second pays d'émigration le plus recherché, l'Angleterre, ce chiffre tombe à 46,0\%). Ce phénomène est moins important chez les hommes où ce chiffre pour les États-Unis est 41,2 \% (32,4\% pour l'Angleterre).

Figure 14. $1^{\text {er }}$ choix du pays de résidence du partenaire sur Shaadi (genre $=$ femme)

Femmes UK: 1er choix pour le pays de résidence du partenaire

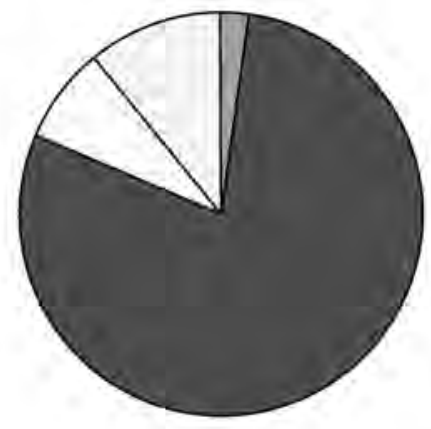

Figure $15.1^{\text {er }}$ choix du pays de résidence du partenaire sur Shaadi (genre $=$ homme)

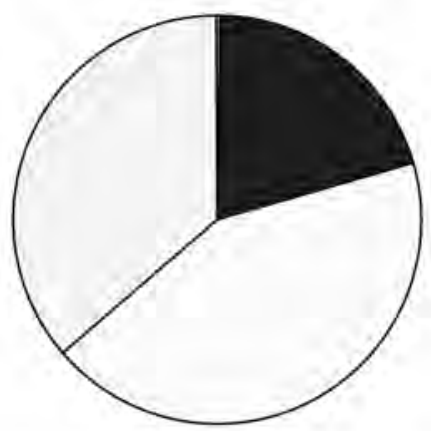


Concluons ici avec une " success story » sur Shaadi qui témoigne des relations entre mariage et mobilité :

Vikram et Divya se sont rencontrés via Shaadi. Vikram relate l'histoire de cette rencontre. Il n'évoque pas la phase de recherche et de consultation des profils et parle immédiatement du premier chat avec Divya au terme duquel ils décident de « se rencontrer en ligne le lendemain ». Puis, ils en viennent à communiquer par téléphone. Vikram sent qu'il devient amoureux mais désire avant tout rencontrer Divya. Ils décident conjointement de se voir en Thaïlande où Divya doit rendre visite à son père. Vikram, quant à lui, vient avec ses parents et sa sœur. Après cela, Vikram n'a plus aucun doute et veut matérialiser son engagement en offrant une bague de fiançailles à Divya. Mais celle-ci ne prévoit de se rendre en Angleterre, où habite Vikram, qu'au mois de juillet. Vikram décide par conséquent de lui faire une surprise en lui rendant visite dans un hôtel en Thaïlande (où elle est allée voir son père) pour la demander en mariage. Suit une période pendant laquelle les futurs mariés continuent à communiquer par téléphone. Enfin, Divya voyage vers l'Angleterre où ils se marient officiellement avant de célébrer l'événement à Jaipur en Inde ${ }^{52}$.

\section{LE WMM - UNE NOUVELLE FORME DE COMMERCE ETHNIQUE}

\section{Le commerce ethnique sur le Web - l'exemple des prestataires de service pour le mariage maghrébin sur le web}

À l'origine de notre recherche, nous étions tentés d'identifier l'économie du WMM à une forme inédite d' ' ethnic business » telle que l'ont thématisé Ivan Light (2000) ou Emmanuel Ma Mung (1992). Le concept d'ethnic business ou d'ethnic entrepreneurship a été développé dans les années 1980 aux ÉtatsUnis ; en France, ce concept renvoie notamment aux travaux d'Emmanuel Ma Mung (dans les années 1990) qui définit le « commerce ethnique » comme suit : « Il s'agit de l'activité pratiquée par des personnes qui utilisent et s'appuient sur des réseaux de solidarité ethnique sur le plan du financement, mais aussi sur le plan de l'approvisionnement, sur celui du recrutement du personnel et parfois même sur celui de l'achalandage lorsque ce commerce vise en premier lieu comme clientèle la communauté dont est issu le commerçant. » Ce que nous retenons, c’est que ce commerce s'appuie sur un réseau préexistant, il est l'activation de ce réseau.

Il existe bien un commerce ethnique du mariage sur le web, mais celui-ci se distingue de ce que nous avons dénommé web matrimonial. Il ne concerne

52. http://www.shaadi.com/shaadi_info/matrimonial_success_stories/index.php. 
pas tant la rencontre, l'appariement des futurs conjoints, que l'organisation du mariage - en tant qu'événement, fête. Prenons l'exemple des prestataires de services pour le mariage maghrébin en France : location de tenues et negafa (habilleuse) ; maquillage au henné ; bijoux ; coiffure ; traiteurs hallal ; pâtisseries ; location et décoration de salle ; animation, musique ; photographes et vidéastes ; location de voiture. Exposer leur offre sur le web, c'est avant tout pour ces prestataires se doter d'une vitrine, se rendre visibles auprès d'un réseau préexistant de clients potentiels.

Figure 16. Orientalement - l' « enclave ethnique » du mariage maghrébin sur le web

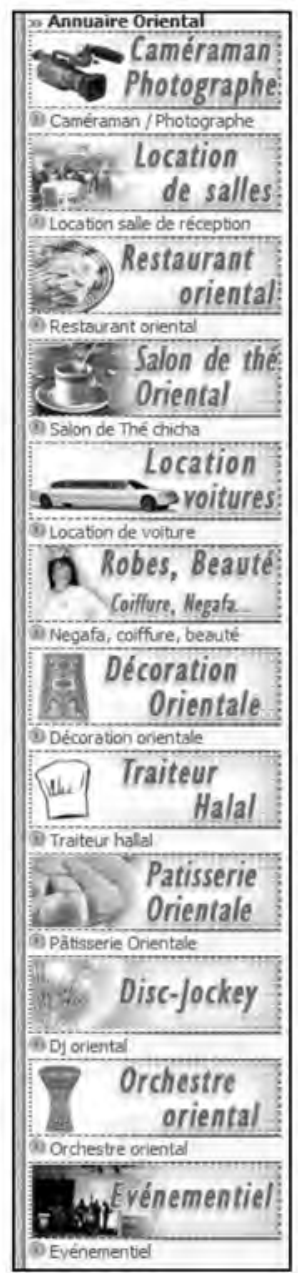

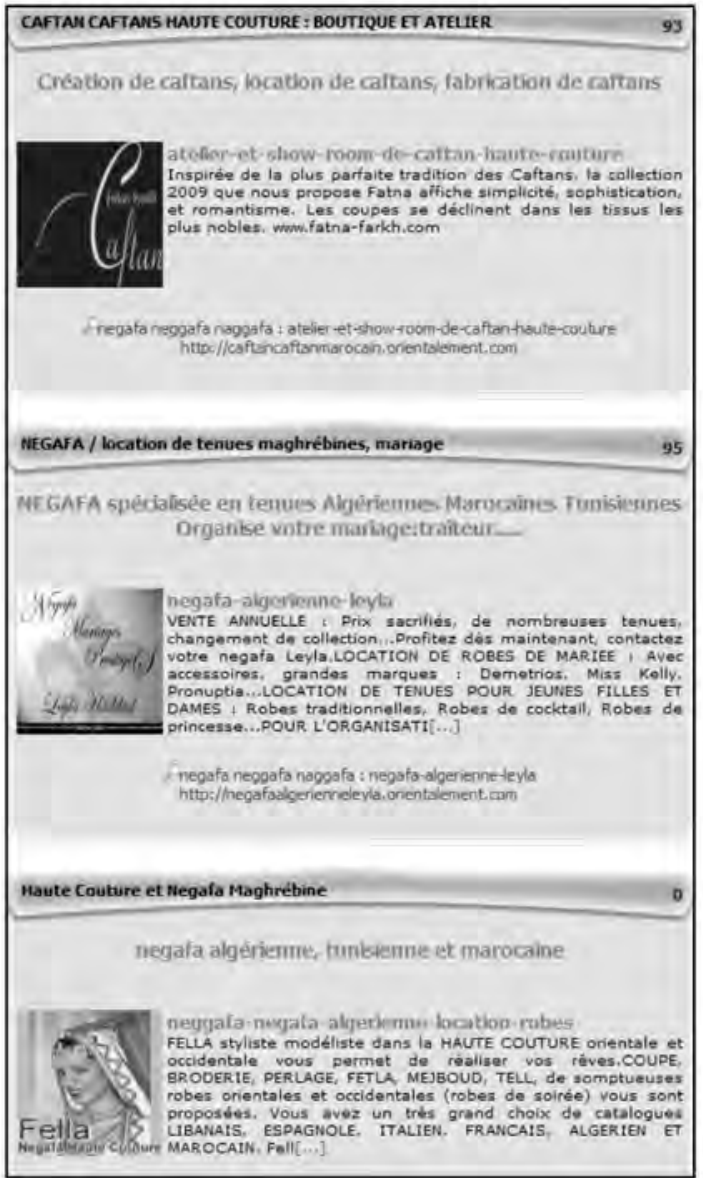


De plus, cette exposition supposant un certain savoir-faire technique, les prestataires font appel à des sociétés de développement web, utilisent des portails de publication d'annonces, etc., qui, cela est décisif, sont spécialisés dans le domaine de l'événementiel maghrébin. Les deux plus importants sont Weboriental ${ }^{53}$ et Orientalement ${ }^{54}$, qui se livrent par ailleurs une rude concurrence. Ces sociétés font plus que rendre possible techniquement la présence online des acteurs du mariage maghrébin. Elles offrent de vastes répertoires de sites, des « annuaires » de la communauté maghrébine, et en ce sens forment d'authentiques espaces pour la « niche ethnique » maghrébine, espaces que viennent occuper les prestataires de services. Il est ainsi possible de parler d'un « virtual estate » répondant au « real estate » (immobilier). Peut-être pourrait-on même découvrir des analogies entre ces espaces et ce qu'on a appelé « enclaves ethniques ». Quoi qu'il en soit, les prestataires de services pour le mariage maghrébin s'appuient sur des réseaux ethniques professionnels. Nous sommes au cœur même de l'entrepreneuriat ethnique.

\section{Le WMM : « faire du commerce » = « faire du réseau »}

Dans le commerce ethnique du mariage maghrébin, à la potentialité du marché répond donc l'actualité du réseau ethnique. L'enjeu du WMM est très différent dans la mesure où la potentialité est tout à la fois celle du marché et celle du réseau : " faire du commerce » et « faire du réseau » s'identifient. En effet, dans le WMM, c'est la " mise en relation » d'individus listés et préalablement non liés entre eux, qui est le moteur de l'économie en question. Ce commerce, dont il faudrait interroger la fonction dans les stratégies de reproduction des communautés ethniques, ne s'identifie pas avec les formes connues du commerce ethnique. Nous sommes en présence d'une nouvelle forme de commerce ethnique.

Ainsi qu'on l'a vu, c'est le profilage ethnique qui est l'instrument premier de ce réseautage entendu comme activité commerciale. Le profilage est la condition du " matching ", celui-ci présidant aux appariements et assurant, formellement ou a priori, la cohérence, l'homogénéité, les affinités au sein du réseau (en devenir). En ce sens, «faire du réseau », c’est aussi «faire de l'ethnie » : ethniciser.

53. www.websurscene.com.

54. www.orientalement.com. 
D'autre part, le WMM ne repose plus sur des « enclaves ethniques », c'est-àdire sur des espaces aux frontières définies. Le WMM crée, en même temps que le réseau, l'espace dans lequel celui-ci pourra s'épanouir. C'est pourquoi mobilité et migration ne doivent plus être considérées comme l' « avant » d'une installation qui sera la condition de possibilité du développement d'un commerce ethnique (au sens traditionnel). Le déplacement des individus est partie intégrante du commerce du WMM lui-même dans la mesure où « faire du réseau », c’est aussi en ce cas bouger, naviguer, se déplacer, migrer. 


\section{RÉFÉRENCES}

BANERJEE A., DUFLO E., GHATAK M., LAFORTUNE J (2009), « Marry for what, Caste and Mate Selection in Modern India », MIT Department of Economics, Working Paper No. 09-14.

http://econ.as.nyu.edu/docs/IO/9800/Marry_for_what_April062009.pdf

BOYD D. (2004), « Friendster and Publicly Articulated Social Networks », Conference on Human Factors and Computing Systems (CHI 2004), Vienna, ACM, April 24-29, 2004.

http://www.danah.org/papers/CHI2004Friendster.pdf

BOYD D. (2005), « Broken Metaphors : Blogging as Liminal Practice ». Media Ecology Association Conference (MEA 2005). New York, June 22-26, 2005. www.danah. org/papers/MEABrokenMetaphors.pdf

BOYD D., ELLISON N. B. (2007), « Social network sites : Definition, history, and scholarship », Journal of Computer-Mediated Communication, 13(1), article 11.

http://jcmc.indiana.edu/vol13/issue1/boyd.ellison.html

CARDON D. (2008), « Le design de la visibilité : un essai de typologie du web 2.0 », Réseaux, 26/152, 2008, pp. 93-137. http://www.internetactu.net/2008/02/01/ le-design-de-la-visibilite-un-essai-de-typologie-du-web-20/

DIMINESCU D. (2005), « Le migrant connecté : pour un manifeste épistémologique », Migrations Société, vol. XVII, n 102, novembre-décembre, pp. 275-293

DIMINESCU D., RENAULT M., GANGLOFF S., PICARD M.A., D’IRIBARNE C., HASSANE S., " Migrants and their use of online technology, Rapport studies from France », (2009) http://ipts.jrc.ec.europa.eu/publications/pub.cfm?id=2460

DOUPLITZKI K. (2009), « Le commerce du moi, modèle économique du profilage », Hermès, 53, « Traçabilité et réseaux ».

HITSCH G. J., HORTACSU A., ARIELY D. (2005), « What Makes You Click : An Empirical Analyis of Online Dating », MIT Sloan Research Paper No. 4603-06

http://www.aeaweb.org/annual_mtg_papers/2006/0106_0800_0502.pdf

ILLOUZ E. (2006), Les sentiments du capitalisme, Paris, Le Seuil. Un extrait a été publié dans Réseaux $n^{\circ}$ 138, «Les blogs », sous le titre « Réseaux amoureux sur Internet » (2006).

LARDELLIER P. (2004), Le cœur Net. Célibat et amours sur le web, Paris, Belin.

LIGHT I., GOLD S. J. (2000), Ethnic Economies, Oxford, Elsevier. 
MA MUNG E. (1992), « L'expansion du commerce ethnique : Asiatiques et Maghrébins dans la région parisienne ", Revue européenne des migrations internationales, vol. 8, $\mathrm{n}^{\circ} 1,1992$.

http://www.persee.fr/web/revues/home/prescript/article/remi_0765-0752_1992_ num_8_1_1593

PACHE-HUBER V. (2004), Le mariage de l'Amour et de la Raison. Stratégies matrimoniales de la classe moyenne en Inde, Münster/Hambourg/Berlin/Vienne/Londres, LIT Verlag.

PALRIWALA R., UBEROI P. (eds.) (2008), Marriage, migration and Gender, New Dehli, Sage.

PORTES A., HALLER W., GUARNIZO L. (2002), « Transnational Entrepreneurs : The Emergence and Determinants of an Alternative Form of Immigrant Economic Adaptation », American Sociological Review, 67 (2).

STIEGLER B., Ars Industrialis (2006), Réenchanter le monde. La valeur esprit contre le populisme industriel, Paris, Flammarion.

TISSERON S. (2008), Virtuel mon amour : penser, aimer, souffrir à l'ère des nouvelles technologies, Paris, Albin Michel.

TOLENTINO R. B. (1996), « Bodies, Letters, Catalogs : Filipinas in Transnational Space », Social Text, No. 48., pp. 49-76. 